TITLE:

\title{
Evolution of geometric and electronic structure in ultrathin In films on $\mathrm{Cu}(001)$
}

\section{$\operatorname{AUTHOR}(\mathrm{S}):$}

Nakagawa, T; Mitsushima, S; Okuyama, $\mathrm{H}$; Nishijima, M; Aruga, T

\section{CITATION:}

Nakagawa, T ...[et al]. Evolution of geometric and electronic structure in ultrathin In films on Cu(001). PHYSICAL REVIEW B 2002, 66(8): 085402.

ISSUE DATE:

2002-08-15

URL:

http://hdl.handle.net/2433/50055

RIGHT:

Copyright 2002 American Physical Society 
PHYSICAL REVIEW B 66, 085402 (2002)

\title{
Evolution of geometric and electronic structure in ultrathin In films on $\mathrm{Cu}(001)$
}

\author{
T. Nakagawa, S. Mitsushima, H. Okuyama, M. Nishijima, and T. Aruga* \\ Department of Chemistry, Graduate School of Science, Kyoto University, Kyoto 606-8502, Japan
}

(Received 11 December 2001; revised manuscript received 13 May 2002; published 1 August 2002)

\begin{abstract}
We have studied the initial growth and the evolution of the atomic and electronic structure of ultrathin In films on $\mathrm{Cu}(001)$ at $110-300 \mathrm{~K}$ by scanning-tunneling microscopy, low-energy electron diffraction, Augerelectron spectroscopy, work-function measurement, and angle-resolved ultraviolet photoelectron spectroscopy. While the deposition at $110-200 \mathrm{~K}$ results in the In adsorption on $\mathrm{Cu}(001)$ terraces, In atoms deposited at 300 $\mathrm{K}$ are initially incorporated into the topmost layer of the substrate. At coverages between 0.26 and $0.35 \mathrm{ML}$ ( $1 \mathrm{ML}=1.53 \times 10^{15} \mathrm{~cm}^{-2}$ ), surface dealloying occurs and $\mathrm{In}$ atoms form an overlayer on top of the $\mathrm{Cu}(001)$ terrace. A variety of phases, including seven long-range-ordered ones, are formed depending on the deposition temperature $(110-300 \mathrm{~K})$ and the In coverage $(0-1 \mathrm{ML})$. When In is deposited at 110-200 K, the surface shows two superstructures, which are metastable and transform irreversibly to other phases upon annealing. When $\mathrm{In}$ is deposited onto $\mathrm{Cu}(001)$ at $300 \mathrm{~K}$, the surface exhibits three distinct superstructures different from those obtained at 110-200 K, and two of them undergo reversible phase transitions upon the temperature increase. For In deposition at $300 \mathrm{~K}$, a surface resonance band is observed, whose intensity is highest around the completion of a monolayer, indicating that the resonance has a large amplitude at the In-Cu interface. Coverage-dependent photoemission data show that the resonance splits off from the $\mathrm{Cu} 4 s p$ band at $\sim 0.3 \mathrm{ML}$ and shows a nearly free electron like dispersion similar to that of the $\mathrm{Cu} 4 s p$ band. A similar interface resonance state is observed for the surface grown at $110 \mathrm{~K}$, while the dispersion is modulated due to the unmatched interface structure. The mechanism of formation of the surface resonance and its relevance to the evolution of the geometric structure is discussed.
\end{abstract}

DOI: 10.1103/PhysRevB.66.085402

PACS number(s): 73.20.At, 68.35.Bs, 71.45.Lr

\section{INTRODUCTION}

The electronic surface states in ultrathin metal films on metal substrates and their induced properties have been attracting increasing attention in recent years. ${ }^{1}$ In particular, the discovery of the surface charge-density-wave (CDW) states on metals ${ }^{2,3}$ indicated the possibility that metal surfaces serve as a new field of the physics of low-dimensional materials. On surfaces, the scanning-probe microscopies provide local information of CDWs and associated lattice distortions, which is usually inaccessible for bulk materials. The interplay of the surface electronic states (surface resonances) with the bulk electronic states of metal substrates would cause substantial differences with the cases of bulk materials and semiconductor surfaces. We reported that the Fermi surface nesting for an adsorbate-induced surface-resonance band drives an order-order structural phase transition in the In monolayer on $\mathrm{Cu}(001){ }^{2}$ In the present paper we give a comprehensive account of the initial growth process of In on $\mathrm{Cu}(001)$ in both atomic and electronic aspects.

In, with a $4 d^{10} 5 s^{2} 5 p^{1}$ valence configuration, has a low melting temperature, $T_{m}=430 \mathrm{~K}$, indicating a relatively low cohesive energy, and a face-centered tetragonal structure with $a=b=4.59 \AA$ and $c=4.95 \AA$, ${ }^{4}$ which can be regarded as a deformed fcc structure. The large lattice mismatch $(\sim 30 \%)$ between $\mathrm{Cu}(3.62 \AA)$ and In plays a role in the initial growth as well as in the formation of ordered phases as discussed below. There is also a large surface energy difference between $\mathrm{Cu}\left(2.0 \mathrm{~J} \mathrm{~m}^{-2}\right)$ and $\mathrm{In}\left(0.7 \mathrm{~J} \mathrm{~m}^{-2}\right),{ }^{5}$ which may suggest that deposited In wets the $\mathrm{Cu}$ surface rather than forming 3D islands. $\mathrm{Cu}$ and $\mathrm{In}$ are known to be miscible in the bulk, and have some alloy phases. ${ }^{6}$
During the last decade, intensive studies with scanning tunneling microscopy (STM) have revised the studies of thin-film growth. In particular, the alloy formation at initial growth stages is proven for many metal-on-metal systems. ${ }^{7-12}$ It will be shown below that in the present case the deposited In atoms initially substitute with $\mathrm{Cu}$ atoms in the topmost layer but, with increasing coverage, dealloying transition occurs which results in an In overlayer on top of the $\mathrm{Cu}$ terrace. At higher coverages, a variety of phases are formed according to coverage and temperature, which are accessed by STM and low-energy electron diffraction (LEED). From the electronic side, the evolution of twodimensional surface electronic structures on metal surfaces remains unclear in most systems. Angle-resolved ultraviolet photoelectron spectroscopy (ARUPS) probes the twodimensional band structure of the surfaces, and has been applied extensively to the study of, for instance, semiconductor surfaces. For metal-on-metal systems, several extensive works have been done for the alkali-metal adsorption on metal surfaces, ${ }^{13-17}$ which in the present context is considered to be a prototypical system for the modified metal surfaces. In contrast to semiconductor surfaces, however, the complexity of substrate bands near the Fermi level and the hybridization of adsorbate and substrate bands which results in broad resonance states tends to make the interpretation of the data ambiguous. Thus a lot of aspects are still left unknown about how the surface electronic states evolve in the formation of metal-metal interfaces, which is desirable to get a clue for the search of new surface materials with novel properties.

This paper is constructed as follows. After the experimental details are described in Sec. II, the results of AES, LEED, 
work-function measurement, STM, and ARUPS for the In growth at 110, 200, and $300 \mathrm{~K}$ are shown in Sec. III A. The initial In deposition at $110 \mathrm{~K}$ results in isolated In adatoms scattered on the surface due to the limited mobility. At 200 $\mathrm{K}$, In adatoms form two-dimensional islands. At $300 \mathrm{~K}$, initial In deposition leads to the incorporation of In atoms into the topmost layer. Upon further In deposition, a dealloying transition occurs which leads to an In overlayer on top of the $\mathrm{Cu}$ surface. In Sec. III B, we summarize a variety of phases observed by LEED and STM as a function of temperature and coverage. In Sec. III C, we describe the ARUPS results for the evolution of electronic surface resonance on In/ $\mathrm{Cu}(001)$. At $300 \mathrm{~K}$, the In-induced resonance appears at the coverage corresponding to the dealloying transition and shows significant dispersion even in the disordered phase at low coverages. The dispersion of the surface resonance band mimics the edge of the projected $\mathrm{Cu}$ bulk bands. On the other hand, the resonance band observed at $110 \mathrm{~K}$ shows a different dispersion. In Sec. IV, we discuss on the growth of In on $\mathrm{Cu}(001)$. After the absolute coverage calibration for In is given based on the compilation of different experimental data, the phase diagram for the $\mathrm{In} / \mathrm{Cu}(001)$ system is deduced. The detailed account is given for the alloyingdealloying transition observed in submonolayer coverages. The nature of the electronic surface resonance is discussed in terms of the bond formation at the $\mathrm{Cu}$-In interface.

\section{EXPERIMENT}

STM and ARUPS experiments were performed in different chambers. The operating pressures were $5 \times 10^{-11}$ and $1 \times 10^{-10}$ Torr in the STM and ARUPS chambers, respectively. Both chambers are equipped with LEED optics, which were used also as retarding field analyzers for AES, and ion sputtering facilities. A variable-temperature STM (Oxford Instruments) was used to obtain images at sample temperatures from 100 to $450 \mathrm{~K}$. All the STM images shown below were taken in the constant-current (topographic) mode. For wide scan STM images, bias voltages between 300 and $500 \mathrm{mV}$ and tunneling currents between 1 and $2 \mathrm{nA}$, corresponding to the tunneling gap resistance of 100-500 $\mathrm{M} \Omega$, were used. For high-resolution images, the gap resistance was decreased to $0.2-5 \mathrm{M} \Omega$ so that the tip-surface distance gets closer. The ARUPS experiments were done with ARUPS10 (VG Microtech). Unpolarized He I and He II radiation were used. The energy resolution of the analyzer was set at $60 \mathrm{meV}$ and the angle of acceptance at $1.0^{\circ}$. The systematic error in the determination of peak positions was estimated to be $<5 \mathrm{meV}$. The In $4 d$ core-level photoelectron spectra were measured by using He II, with the light incidence normal to the surface and the emission angle of $45^{\circ}$. The work function was determined from the width of photoelectron spectra with the sample biased at $-6.30 \mathrm{~V}$. The experimental error in the work-function measurement was within $20 \mathrm{meV}$.

$\mathrm{The} \mathrm{Cu}(001)$ crystals were mechanically and electrochemically polished and then cleaned in ultrahigh vacuum by repeated cycles of Ar ion sputtering and annealing at 700$770 \mathrm{~K}$. The cleanness of the samples was checked by AES, the LEED observation of the sharp patterns of the clean and
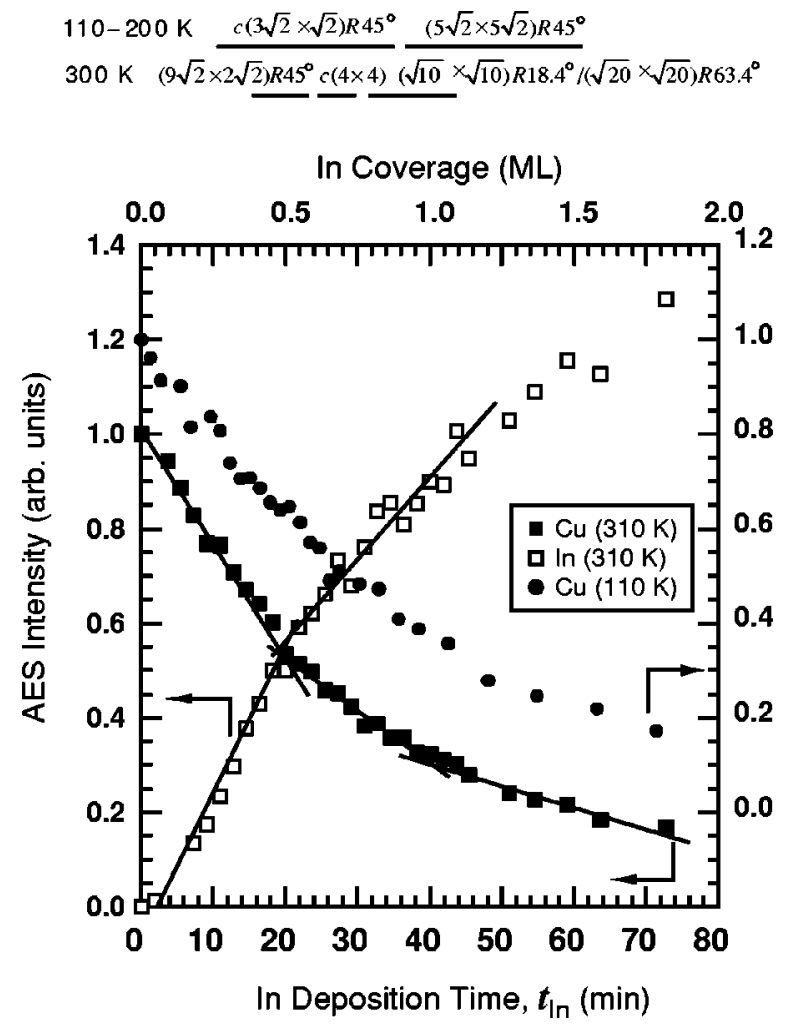

FIG. 1. $\mathrm{Cu} M V V$ AES intensity as a function of In deposition time, $t_{\text {In }}$, for deposition at 110 and $310 \mathrm{~K}$. In $L M M$ AES intensity is also shown for deposition at $310 \mathrm{~K}$. Note that the data for $110 \mathrm{~K}$ are shifted upward. The coverage regions where LEED patterns are observed are also indicated.

In-covered surfaces, the ARUPS observation of the $\mathrm{Cu}(001)$ surface states at $\bar{M}$ (Refs. 18-21) and $\bar{X},{ }^{22}$ and the STM observation of no impurities. In was evaporated from Knudsen-cell-type alumina crucibles that were carefully outgassed before the use. The In coverage was monitored by the work-function measurement and AES. To specify the amount of deposited In, the In deposition time, $t_{\mathrm{In}}$, and the coverage, $\Theta_{\text {In }}$, are used below, where $1 \mathrm{ML}$ is defined as the number density of $\mathrm{Cu}$ atoms on the (001) surface. As discussed in Sec. IV A, the In uptake rate was determined to be 0.025 $\pm 0.001 \mathrm{ML} / \mathrm{min}$ based on the direct counting of the number of In adatoms per unit area.

\section{RESULTS}

\section{A. Initial growth}

\section{AES}

In this section, we show the experimental results relevant to the initial growth mechanism of $\mathrm{In}$ on $\mathrm{Cu}(001)$. Let us begin with the AES uptake curve.

Figure 1 shows the variation of the $\mathrm{Cu} M V V(60 \mathrm{eV}) \mathrm{AES}$ intensity as a function of the In deposition time, $t_{\mathrm{In}}$, where the sample was maintained at 110 and $310 \mathrm{~K}$ during the deposition. For the deposition at $310 \mathrm{~K}$, the variation of In LMM $(402 \mathrm{eV})$ is also shown. (For $110 \mathrm{~K}$, In AES was not measured in order to avoid contamination.) 
For the deposition at $310 \mathrm{~K}$, simultaneous breaks are observed for both the $\mathrm{Cu}$ and In curves at $t_{\mathrm{In}}=20 \mathrm{~min}\left(\Theta_{\text {In }}\right.$ $=0.50 \mathrm{ML}$ ), and less significant ones around $t_{\text {In }}$ $=40 \mathrm{~min}\left(\Theta_{\mathrm{In}}=1.0 \mathrm{ML}\right)$. The first break points occur when the $\mathrm{Cu}$ signal is attenuated to 0.54 of the initial value. The second ones correspond to the $\mathrm{Cu}$ signal attenuation by a factor of $0.30\left(\sim 0.54^{2}\right)$. This appears to be consistent with the layer-by-layer growth of In on top of $\mathrm{Cu}(001)$.

The behavior of the $\mathrm{Cu}$ AES intensity at $110 \mathrm{~K}$ is qualitatively similar to that taken at $300 \mathrm{~K}$ as shown in Fig. 1 . This indicates that the 3D island growth of In is hindered also at $110 \mathrm{~K}$. At $110 \mathrm{~K}$, however, the breaks are not significant and the attenuation of the Cu AES intensity appears to be consistent with the continuous, exponential decay, indicating that the growth mode may slightly deviate from the ideal layer-by-layer growth

While no considerable difference is found in the AES uptake curves at the initial stage $(<0.5 \mathrm{ML})$, the microscopic growth mechanism up to the completion of the first ordered layer depends strongly on the sample temperature during the In deposition, as will be shown in the following sections.

\section{STM measurement at $200 \mathrm{~K}$}

In this and the following subsections, the STM results for the growth of In at sample temperatures of 200 and $300 \mathrm{~K}$, respectively, are summarized. The growth at temperatures lower than $200 \mathrm{~K}$ was not studied by STM due to an instrumental limitation.

Figure 2 shows the STM images taken at $130 \mathrm{~K}$ after In deposition at $200 \mathrm{~K}$. The STM image taken at $\Theta_{\text {In }}$ $=0.03 \mathrm{ML}$ is shown in Fig. 2(a) and Fig. 2(c). The bright dots condensed at step edges have an apparent height of $\sim 2.6 \AA$, which is larger than that of monolayer steps on $\mathrm{Cu}(001), 1.8 \AA$, and are interpreted as In atoms. These images indicate that In atoms initially nucleate on terraces and at step edges. This suggests the limited mobility of In on terraces at $200 \mathrm{~K}$, while the diffusion along steps is fast as indicated by the complete decoration of steps at this coverage. It is noted that a small amount of isolated In atoms is found inside terraces. The apparent height difference between these bright $\mathrm{In}$ atoms and the surrounding $\mathrm{Cu}$ terrace is only $0.3-0.5 \AA$, which suggests that the bright atoms are not In atoms on top of the terrace but the ones incorporated into the topmost layer of the substrate. The incorporated In atoms are found within the vicinity $(\sim 20 \AA)$ of steps in both the upper and lower terraces, indicating that the incorporation of In is caused only by the exchange process at steps. This result is in good agreement with the previous observation. ${ }^{23}$ It should be noted that the exchange process at steps are sometimes supposed to take place between an adatom on the lower terrace and an atom in the upper terrace, which results in the incorporation of the foreign atom only in the upper terrace. The observation of incorporated atoms in the lower terrace suggests that another exchange process is operating, in which an atom in the lower terrace is involved. This process might be similar to that found for Re on $\operatorname{Ir}(001){ }^{24}$

Figure 2(b) shows an atomic-resolution STM image near a step for $\Theta_{\text {In }}=0.08 \mathrm{ML}$. The $1 \times 1$ terrace with In atoms
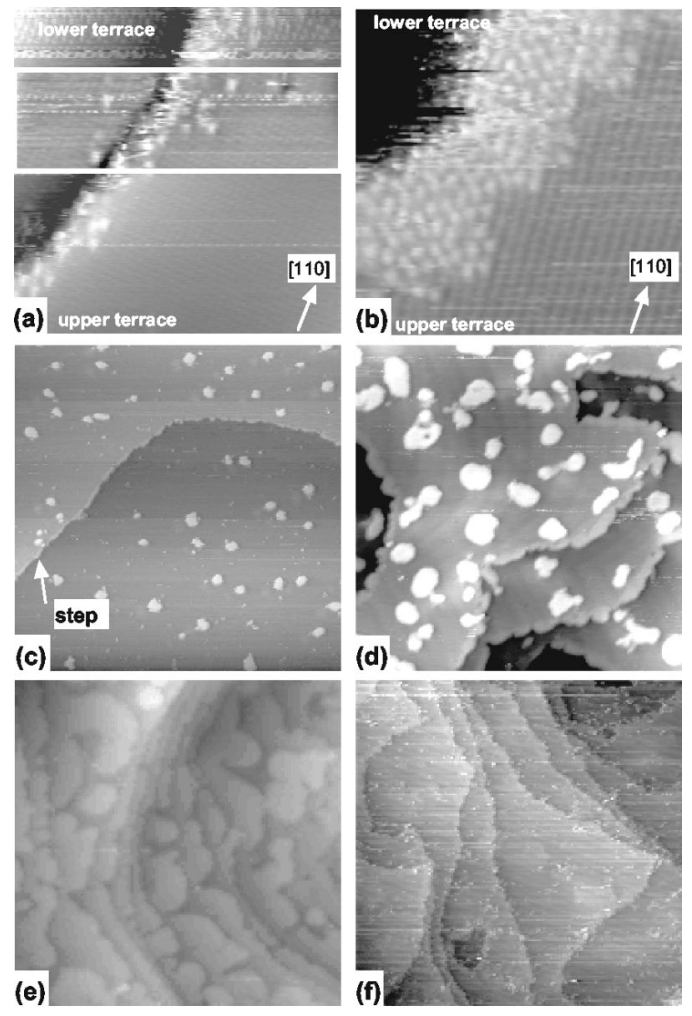

FIG. 2. Atomically resolved STM images taken at $130 \mathrm{~K}$ after In deposition at $200 \mathrm{~K}$ for (a) $\Theta_{\text {In }}=0.03 \mathrm{ML}\left(90 \times 90 \AA^{2}, V_{s}\right.$ $=9 \mathrm{mV}, I_{t}=35 \mathrm{nA}$ ) with the middle part high-pass filtered to show incorporated In atoms in both the upper and lower terrace, and (b) $\Theta_{\mathrm{In}}=0.08 \mathrm{ML}$ at $200 \mathrm{~K} \quad\left(65 \times 65 \AA^{2}, V_{s}=9 \mathrm{mV}, \quad I_{t}\right.$ $=10 \mathrm{nA}$ ). Wide-scan STM images taken at $130 \mathrm{~K}$ after In deposition at $200 \mathrm{~K}$ for: (c) $\Theta_{\text {In }}=0.03 \mathrm{ML}\left(1000 \times 1000 \AA^{2}\right)$; (d) $\Theta_{\text {In }}$ $=0.15 \mathrm{ML}\left(1500 \times 1500 \AA^{2}\right) ; \quad$ (e) $\quad \Theta_{\text {In }}=0.45 \mathrm{ML}(1500$ $\left.\times 1500 \AA^{2}\right) ;(f) \Theta_{\text {In }}=0.88 \mathrm{ML}\left(950 \times 950 \AA^{2}\right)$.

condensed at the step is observed. The average distance between neighboring In atoms is $\sim 3.3 \AA$, which is close to the nearest-neighbor distance in bulk In, $3.25 \AA$. However, any ordered structure does not appear at this coverage.

Figure 2(d) shows a wide-scan STM image for $\Theta_{\text {In }}$ $=0.15 \mathrm{ML}$. The steps are completely decorated with $2 \mathrm{D}$ In islands. It is also found that 2D islands with sizes of 50-100 $\AA$ are formed on terraces. The apparent height of the $2 \mathrm{D}$ islands are $\sim 2.6 \AA$, which is the same as that for In atoms condensed at steps. Although the incorporation is possible at steps, the rate of incorporation is very low at $200 \mathrm{~K}$. Figure 2 (e) shows a wide-scan STM image for $\Theta_{\text {In }}=0.45$ ML. The $2 \mathrm{D}$ islands are completely percolated while no $3 \mathrm{D}$ islands are formed.

As shown later, the first In layer is completed at $\Theta_{\text {In }}$ $=0.5 \mathrm{ML}$. The wide-scan STM image for $\Theta_{\text {In }}=0.88 \mathrm{ML}$ [Fig. 2(f)] indicates that the surface is free from 3D islands. Note also that few 2D islands are observed on terraces, which suggests that the 2D In island growth is hindered on top of the In layer because of the low migration energy of In on In. ${ }^{25}$ Thus In grows on $\mathrm{Cu}(001)$ surface according essentially to the step-flow growth mode at $200 \mathrm{~K}$ after the first layer is completed. 

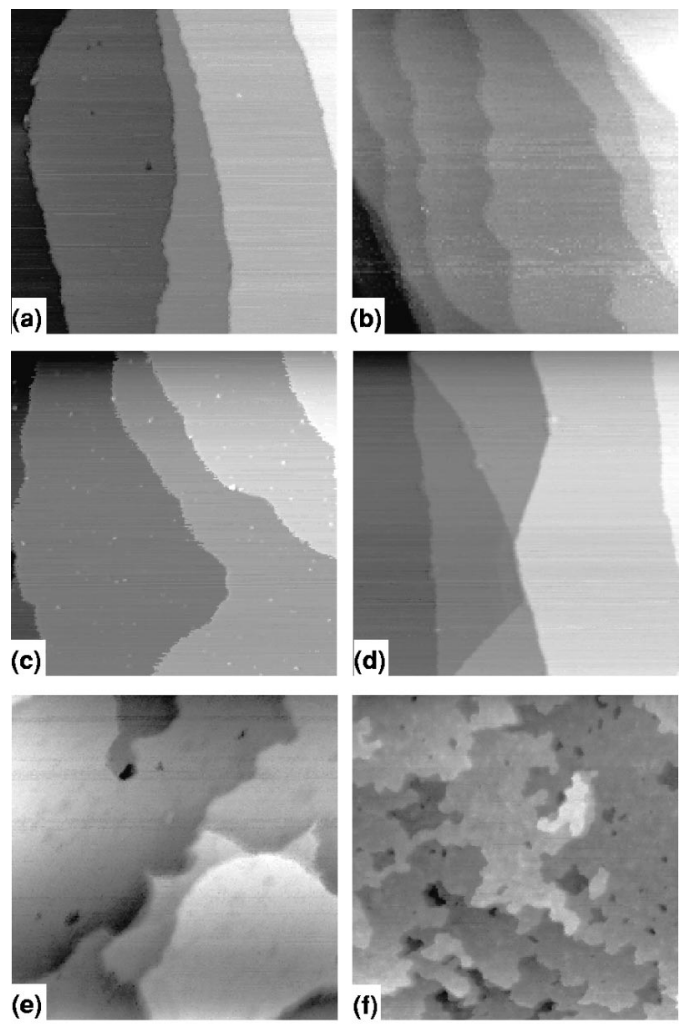

FIG. 3. (a) Wide-scan STM image of clean $\mathrm{Cu}(001)-(1$ $\times 1)\left(1100 \times 1100 \AA^{2}\right)$. (b) $-($ f) Wide-scan STM images taken at $300 \mathrm{~K}$ after In deposition at $300 \mathrm{~K}$ for (b) $\Theta_{\text {In }}=0.13 \mathrm{ML}(1800$ $\left.\times 1800 \AA^{2}\right) ; \quad$ (c) $\Theta_{\text {In }}=0.50 \mathrm{ML} \quad\left(1500 \times 1500 \AA^{2}\right) ;$ (d) $\Theta_{\text {In }}$ $=0.65 \mathrm{ML}\left(1500 \times 1500 \AA^{2}\right) ;(\mathrm{e}) \Theta_{\mathrm{In}}=1.0 \mathrm{ML}\left(930 \times 930 \AA^{2}\right)$; (f) $\Theta_{\text {In }}=2.5 \mathrm{ML}\left(1500 \times 1500 \AA^{2}\right)$.

\section{STM measurement at $300 \mathrm{~K}$}

The STM images for the $\mathrm{Cu}(001)$ surface deposited with In at $300 \mathrm{~K}$ are shown in Figs. 3 and 4. The STM image of the clean $\mathrm{Cu}(001)$ surface [Fig. 3(a)] exhibits wide terraces $\left(\begin{array}{c}\sim 00 \AA \\ \AA\end{array}\right)$ and nearly straight steps. The high-resolution image [Fig. 4(a)] shows the $1 \times 1$ structure with a lattice constant of $a_{0}=2.56 \AA$ and an apparent corrugation amplitude of $0.1-0.3 \AA$. The height of the steps on $\mathrm{Cu}(001)-1 \times 1$ is 1.8 $\AA$, which we used for the calibration of the height.

Figure 3(b) shows a wide-scan STM image for $\Theta_{\text {In }}$ $=0.13$ ML. No isolated adatoms or islands are observed on terraces. While the steps appear to be wavy as compared with those on the clean surface, the contrast difference observed at original step positions at $200 \mathrm{~K}$ [Fig. 2(d)] is not observed (i.e., the height of steps is $1.8 \AA$ ), indicating that the wavy steps are not due to the In condensation. In a highresolution image [Fig. 4(b)], we observe many bright protrusions, which are located on the $(1 \times 1)$ lattice positions and have an apparent height higher than surrounding $\mathrm{Cu}$ atoms by $0.3-0.5 \AA$. We assigned these bright protrusions to the In atoms embedded in the topmost surface layer. The embedded In atoms are scattered on the surface and no significant density differences were observed depending on the distance from steps.

As is seen in Fig. 4(b), the high-resolution images for the In-incorporated surface are associated with considerable

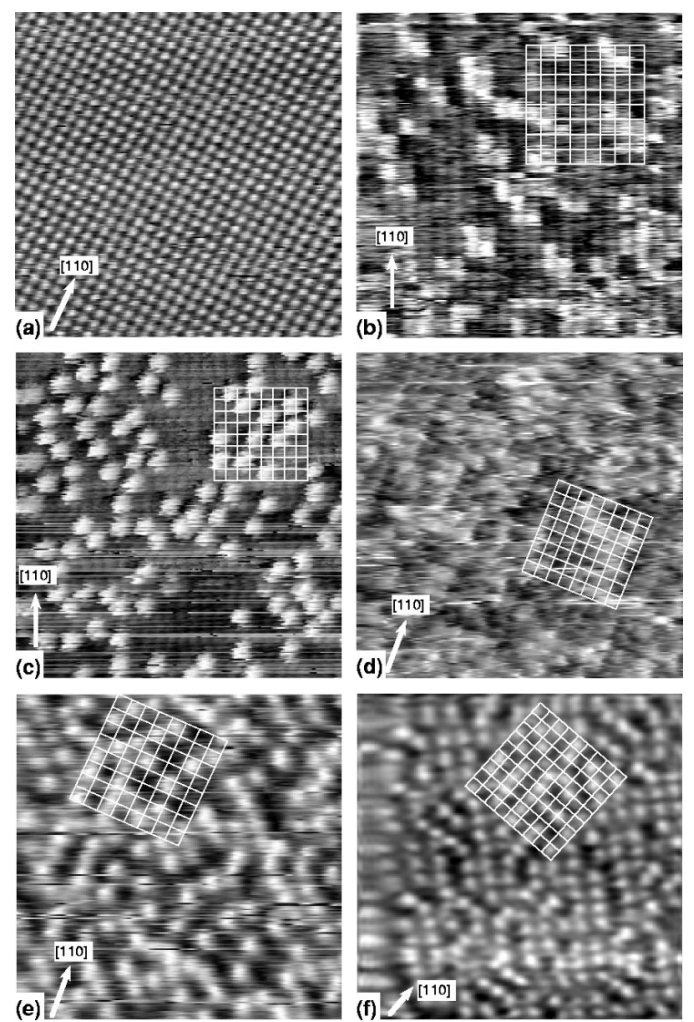

FIG. 4. (a) Atomically resolved STM image of clean $\mathrm{Cu}(001)-(1 \times 1) \quad\left(95 \times 95 \AA^{2}, V_{s}=8 \mathrm{mV}, I_{t}=6 \mathrm{nA}\right) . \quad(\mathrm{b})-(\mathrm{f})$ Atomically resolved STM image of $\mathrm{Cu}(001)$ after In deposition at $300 \mathrm{~K}$ [grids indicate the $\mathrm{Cu}(001)-1 \times 1$ lattice]: (b) $\Theta_{\mathrm{In}}=0.13$ $\pm 0.03 \mathrm{ML}$ taken at $T=300 \mathrm{~K} \quad\left(53 \times 53 \AA^{2}, V_{s}=5 \mathrm{mV}, I_{t}\right.$ $=30 \mathrm{nA}) ;\left(\right.$ c) $\Theta_{\mathrm{In}}=0.13 \pm 0.03 \mathrm{ML}$ taken at $T=130 \mathrm{~K} \quad(70$ $\left.\times 70 \AA^{2}, V_{s}=-100 \mathrm{mV}, I_{t}=20 \mathrm{nA}\right) ;(\mathrm{d}) \Theta_{\mathrm{In}}=0.26 \pm 0.05 \mathrm{ML}$ taken at $300 \mathrm{~K}\left(67 \times 67 \AA^{2}, V_{s}=-4 \mathrm{mV}, I_{t}=17 \mathrm{nA}\right)$; (e) $\Theta_{\text {In }}$ $=0.37 \pm 0.05 \mathrm{ML}$ taken at $300 \mathrm{~K}\left(57 \times 57 \AA^{2}, V_{s}=-6 \mathrm{mV}, I_{t}\right.$ $=24 \mathrm{nA}) ;$ (f) $\Theta_{\text {In }}=0.37 \pm 0.05 \mathrm{ML}$ taken at $300 \mathrm{~K} \quad(67$ $\left.\times 67 \AA^{2}, V_{s}=9 \mathrm{mV}, I_{t}=40 \mathrm{nA}\right)$.

horizontal noises, and some of the embedded In atoms are imaged as the bars elongated along [110] direction. This is due to the diffusion of the embedded In atoms ${ }^{23}$ as can be observed by time evolution of high-resolution images (not shown).

Figure 4(c) shows a high-resolution image for $\Theta_{\text {In }}$ $=0.13 \mathrm{ML}$ measured after the surface was quenched to 130 $\mathrm{K}$ in order to reduce the mobility. The embedded In atoms are imaged as round-shaped protrusions with small noises, indicating that the embedded In atoms do not significantly move at this temperature.

One may want to compare the apparent height difference, 0.3-0.5 $\AA$, observed here with the perpendicular shift of In atoms embedded in $\mathrm{Cu}(001), 0.45 \pm 0.05 \AA$, deduced by the analysis of low-energy ion scattering data. ${ }^{26}$ It is noted, however, that the apparent height observed in STM cannot be directly related to the real atomic positions. Actually, the height (contrast) difference between embedded In and surrounding $\mathrm{Cu}$ is observed only with low bias voltages and high tunneling currents, which correspond to small tipsurface distances. The STM images for the surface of Figs. 


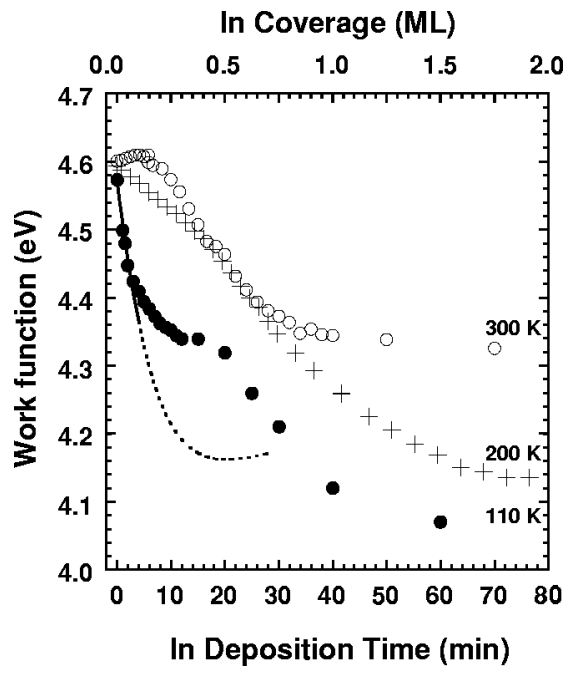

FIG. 5. Work-function change at 110,200 , and $300 \mathrm{~K}$ as a function of deposition time, $t_{\text {In }}$. The curve shows the result of the fitting of the Topping's formula to the initial decrease in the $110 \mathrm{~K}$ data (see text).

4(b) and 4(c) measured at sample bias of $\sim 300 \mathrm{mV}$ and tunneling current of $1 \mathrm{nA}$ show no contrast difference between In and $\mathrm{Cu}$. The difference in the image contrast between different metal elements has been previously attributed to several mechanisms. ${ }^{27-29}$ In our case, the chemical contrast is reproducibly observed and does not require any special tip condition, which excludes the role of impurity atoms at tip apex. Since the work-function change induced by small amounts of In atoms is negligible as shown later (see Fig. 5), at least the electrostatic part of the barrier height should be negligible to explain the chemical contrast. While $\mathrm{Cu}(001)$ has a Schockley type surface state near the Fermi level, ${ }^{22}$ the chemical contrast is observed both at negative and positive bias voltages, which excludes the possibility that the surface state induces the chemical contrast. We therefore consider that the chemical contrast observed here is due to the difference in the density of state near the Fermi level in the tip-surface systems.

An atomically resolved STM image of the surface at $\Theta_{\text {In }}=0.26 \mathrm{ML}$ is shown in Fig. 4(d). There exist many bright protrusions together with some dark ones (see the upper-left corner). Both types of protrusions are aligned on the $1 \times 1$ mesh. Although the $1 \times 1$ structure due to $\mathrm{Cu}$ atoms is blurred compared to the surface for $\Theta_{\text {In }}=0.13 \mathrm{ML}$ [Fig. 4(b)], the image indicates that the top layer is composed of a $1 \times 1 \mathrm{Cu}$ layer embedded with In atoms. Another STM image for the same surface with a different tunneling condition is shown in Fig. 4(e), in which In atoms are clearly imaged though $\mathrm{Cu}$ atoms are hardly recognized. All the In atoms are located on the $1 \times 1$ lattice positions as shown by the grid in Fig. 4(e). Two types of local order of In atoms are recognized: the In atoms are arranged in a $c(2 \times 2)$ symmetry or form close-packed chains along [110] with a nearestneighbor spacing of $\sim 2.6 \AA$. Although the surface alloy is confined in the $1 \times 1$ structure, it is likely that the surface stress increases greatly due to the large size mismatch.
The surface exhibits a disordered structure at coverages of $0.3-0.35 \mathrm{ML}$. An STM image taken at $130 \mathrm{~K}$ for $\Theta_{\text {In }}$ $=0.37 \mathrm{ML}$ is shown in Fig. 4(f). We could not get any STM images containing $\mathrm{Cu}$ atoms at this In coverage under any tunneling conditions. The surface appears to be composed of two types of domains with different structure: a squarelike lattice with its sides aligned along [100] and parallel chains along [110]. The distance between the neighboring atoms in the squarelike domains is $\sim 3.6 \AA$, being close to the second-nearest-neighbor distance of the $1 \times 1$ structure $\left(\sqrt{2} a_{0}\right)$, meaning that the squarelike structure forms a local $c(2 \times 2)$ periodicity. In the chain structure, the distance between the nearest-neighbor atoms varies in the range of 3.1$3.8 \AA$, which does not agree with $a_{0}$ or $2 a_{0}$, but is close to the metallic diameter of In, $3.25 \AA$. This indicates that the spacing in the chain structure is determined mainly by the interaction between In atoms. Consequently, the protrusions in the STM images do not agree with the $1 \times 1$ mesh. The surface shows no long-range order, which agrees with the LEED pattern without any superstructure spots but with the high background intensity near half-order positions. The structural features of this surface are similar to those of an ordered structure formed at $\Theta_{\mathrm{In}}=0.5 \mathrm{ML}$ (shown later), and hence they are understood as embryos of the long-range order. From the viewpoint of the initial growth mechanism, it should be emphasized that the random substitutional surface alloy with a $1 \times 1$ average translational symmetry transforms between $\Theta_{\text {In }}=0.26$ and $0.37 \mathrm{ML}$ to the structure in which $\mathrm{Cu}$ atoms are removed and $\mathrm{In}$ atoms do not occupy the $1 \times 1$ lattice sites.

A wide-scan STM image for $\Theta_{\text {In }}=0.5 \mathrm{ML}$ is shown in Fig. 3(c). The surface is flat and no islands are observed. At this In coverage the atoms on the surface are stable, and we can easily obtain atomically resolved STM images at room temperature as shown in a later section. Wide-scan STM images for $\Theta_{\text {In }}=0.65$ and 1.0 ML shown in Figs. 3(d) and 3(e), respectively, also show flat terraces, while steps get wavy at $\Theta_{\text {In }}=1.0$ ML. Figure 3(f) shows an STM image of the surface at $\Theta_{\mathrm{In}}=2.5 \mathrm{ML}$, where steps wander in a length scale of $<100 \AA$. While the average terrace width is still large $(\sim 500 \AA)$, some small 2D islands appear, indicating that the growth mode is gradually switching from the step-flow to $3 \mathrm{D}$ island growth.

\section{Work-function measurement}

Figure 5 shows the work-function variations as a function of $t_{\mathrm{In}}$ at 110,200 , and $300 \mathrm{~K}$. For deposition at $110 \mathrm{~K}$, the work function initially decreases steeply, and then the slope decreases gradually. The curve shows a distinct break at $t_{\text {In }}$ $\sim 20$ min $\left(\Theta_{\text {In }}=0.5 \mathrm{ML}\right)$, beyond which the work function shows again a steep decrease followed by gradual saturation at $4.1 \mathrm{eV}$, which is near the work-function value of polycrystalline In. ${ }^{30}$ The break may correspond to the completion of the first layer.

The initial steep decrease is understood by the image dipole formation due to the charge transfer from In adatoms to the $\mathrm{Cu}$ substrate. The gradual decrease of the slope upon the increase of In coverage is apparently in agreement with the 
depolarization of the induced image dipole due to the electric field formed by the surrounding dipoles. For alkali-metal adsorption, where adatoms are scattered uniformly on the surface, the so-called Topping's depolarization formula, ${ }^{31,32}$

$$
\Delta \phi=\frac{1}{4 \pi \epsilon_{0}} \frac{ \pm 4 \pi \mu_{0} n}{1+9 \alpha n^{3 / 2}},
$$

is known to explain the initial work-function change well. ${ }^{33}$ Here $\epsilon_{0}$ denotes the static dielectric constant of the vacuum, $\mu_{0}$ the initial dipole moment, $\alpha$ the polarizability, and $n$ the density of adsorbates. It is empirically known that the polarizability, $\alpha$, deduced by fitting the equation to the experimental data is almost the same as the atomic polarizability, or smaller by within a factor of $\sim 2$, than the atomic polarizability. ${ }^{33-38}$ The fitting of Eq. (1) by using the atomic polarizability of In, $10.2 \AA^{3}, 39$ to the work-function change for In adsorption at $110 \mathrm{~K}$ yielded the solid line shown in Fig. 5 and the initial dipole moment of $0.42 \mathrm{D}$. While the initial steep slope is well reproduced, the actual workfunction change beyond $t_{\text {In }}>3 \mathrm{~min}\left(\Theta_{\text {In }}>0.08 \mathrm{ML}\right)$ is much more moderate than that expected by Eq. (1). Changing the value of $\alpha$ within a factor of 2 does not considerably reduce the deviation. We suggest that the deviation is due predominantly to the condensation of In atoms on the surface, which is indicated by the LEED observation of an ordered structure, as shown below, at around $t_{\mathrm{In}}=10 \mathrm{~min}$. The condensation of adatoms leads to the direct overlap of the wave functions of neighboring In atoms, which is associated with the reduction of the adatom-substrate charge transfer.

As compared with the result for $110 \mathrm{~K}$, the work-function decrease at $200 \mathrm{~K}$ is more moderate, in particular, at the initial stage. We observed an almost linear increase up to $t_{\mathrm{In}}=20 \mathrm{~min}\left(\Theta_{\mathrm{In}}=0.5 \mathrm{ML}\right)$, which is not consistent with the depolarization picture for uniformly scattered adatoms. As observed by STM (Fig. 2), In atoms deposited at $200 \mathrm{~K}$ form $2 \mathrm{D}$ islands, which suggests that the local environment around an individual In atom is constant irrespective of the total coverage and therefore the dipole moment associated with an In adatom does not change. In this case, the work function should be the average of those for clean $\mathrm{Cu}(001)$, $\phi_{\mathrm{Cu}}$, and the In monolayer, $\phi_{\mathrm{In}}$,

$$
\phi=\frac{\Theta_{\mathrm{In}}}{\Theta_{\mathrm{In}}^{(1)}} \phi_{\mathrm{In}}+\left(1-\frac{\Theta_{\mathrm{In}}}{\Theta_{\mathrm{In}}^{(1)}}\right) \phi_{\mathrm{Cu}},
$$

where $\Theta_{\text {In }}^{(1)}$ denotes the In coverage at the completion of the first layer. This is in good agreement with the work-function change up to $t_{\text {In }} \sim 20 \min \left(\Theta_{\text {In }} \sim 0.5 \mathrm{ML}\right)$. After the break at $t_{\mathrm{In}} \sim 20 \mathrm{~min}\left(\Theta_{\mathrm{In}}=0.5 \mathrm{ML}\right)$, work function decreases monotonically with the slope decreasing gradually, and approaches the saturation value, $\sim 4.1 \mathrm{eV}$.

For In deposition at $300 \mathrm{~K}$, the work function is almost constant up to $t_{\mathrm{In}} \sim 10 \mathrm{~min}$, then decreases linearly to 4.35 $\mathrm{eV}$ with increasing coverage. The initial period where the work function does not change corresponds to the In incorporation into the topmost layer of the substrate as found by STM [Figs. 4(b) and 4(c)]. After the initial period, the work function starts to decrease at $t_{\mathrm{In}} \sim 10 \mathrm{~min}\left(\Theta_{\mathrm{In}} \sim 0.25 \mathrm{ML}\right)$, which coincides with the disappearance of top-layer $\mathrm{Cu}$ atoms in the high-resolution STM images for $\Theta_{\text {In }}=0.35 \mathrm{ML}$ [Fig. 4(f)].

The asymptotic values of the work function are estimated to be 4.0, 4.1, and $4.3 \mathrm{eV}$ for deposition at 110, 200, and 300 $\mathrm{K}$, respectively. The difference in the work function is attributed to the difference in the degree of surface roughness at different deposition temperatures. For higher deposition temperatures, the surface is expected to become flatter, which leads to higher work function.

\section{Influence of In growth onto the intrinsic surface state on $\mathrm{Cu}(001)$}

The surface state on $\mathrm{Cu}(001)$ in the projected bulk band gap around the $\bar{M}$ point has been thoroughly studied previously. Its origin is explained as the $d_{x^{2}-y^{2}}$-derived band pushed out of the bulk continua by the Coulomb repulsion due to excess $s p$ electrons at the surface. ${ }^{18-21}$ [Note that the $x$ and $y$ axes are defined as the nearest-neighbor directions on $\mathrm{Cu}(001)$.] Since this surface state is localized at the topmost layer, it is sensitive to the change of charge density caused by adsorbates, and hence the change of growth modes can be probed by the surface state. ${ }^{40}$

For clean $\mathrm{Cu}(001)$, the surface state at the $\bar{M}$ point is observed at binding energies of $1.78,1.80$, and $1.81 \mathrm{eV}$ relative to the Fermi level at 110, 300, and $400 \mathrm{~K}$, respectively.

In Fig. 6(a) the spectra for the surface state are shown for the surface deposited with In at $110 \mathrm{~K}$. In deposition results in the decrease in intensity and the shift to higher binding energies, reaching $1.83 \mathrm{eV}$ at $\Theta_{\mathrm{In}}=0.25 \mathrm{ML}$, which is ascribed to the local disorder introduced by isolated In adatoms. As shown in Fig. 6(d), the intensity at $\Theta_{\text {In }}$ $=0.25 \mathrm{ML}$ is $\sim 15 \%$ of the initial value, which indicates that In atoms are initially adsorbed randomly as isolated adatoms and gradually agglomerate during deposition to form islands. This agrees with the interpretation of the workfunction change at $110 \mathrm{~K}$.

Upon In deposition at $200 \mathrm{~K}$, a moderate change in the surface state of $\mathrm{Cu}(001)$ is found as shown in Figs. 6(b) and 6(d). The intensity of the surface state decreases almost linearly and disappears at $\Theta_{\text {In }} \sim 0.63 \mathrm{ML}$. The shift of the peak position is very small $(<10 \mathrm{meV})$, which is in accordance with the STM observation of the 2D-island formation on top of the $\mathrm{Cu}(001)$ terrace.

The spectral change upon In deposition at $300 \mathrm{~K}$ is shown in Fig. 6(c) and the intensity of the surface state is plotted in Fig. 6(d), where the result obtained by using the He II radiation is also shown. The agreement between the results obtained by $\mathrm{He} \mathrm{I}$ and $\mathrm{He}$ II indicates that the decay is not due to the final state effect, but reflects the reduction of the density of surface state. The intensity of the surface state decreases steeply with increasing In deposition time at $300 \mathrm{~K}$. The initial slope is almost the same as that for $110 \mathrm{~K}$. While the decrease at $110 \mathrm{~K}$ gets moderate at $\Theta_{\text {In }}>0.13 \mathrm{ML}$, the decrease at $300 \mathrm{~K}$ is almost linear, and the intensity vanishes around $\Theta_{\text {In }}=0.15$ ML. The peak position is shifted to higher binding energies upon In deposition, reaching $1.88 \mathrm{eV}$ at $\Theta_{\text {In }}=0.13 \mathrm{ML}$. These changes indicate that impinging In 


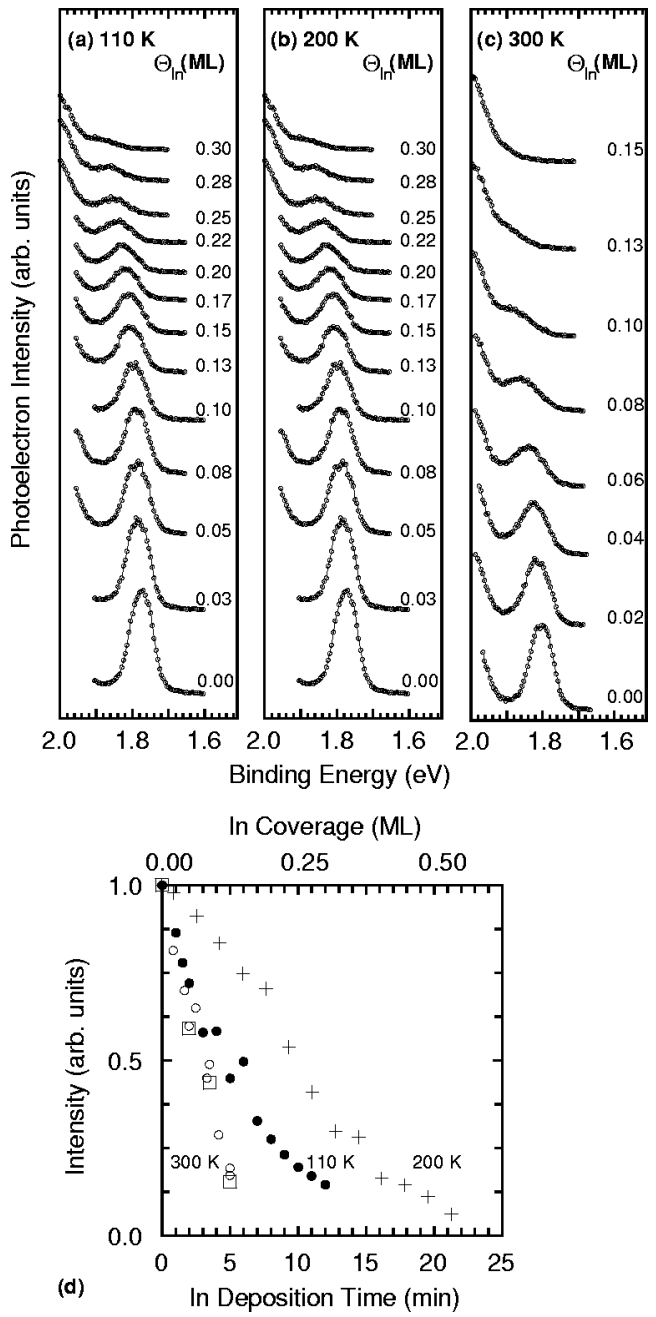

FIG. 6. (a) Angle-resolved photoelectron spectra taken with He-I radiation at $\bar{M}$ for clean and In-covered $\mathrm{Cu}(001)$. The deposition was made at $110 \mathrm{~K}$. (b) The same as (a) except that the deposition and measurement were made at $200 \mathrm{~K}$. (c) The same as (a) except that the deposition and measurement were made at $300 \mathrm{~K}$. (d) The intensities of $\mathrm{Cu} 3 d$ Tamm state peak measured with He-I radiation at different deposition temperatures: $110 \mathrm{~K}$ (filled circles); $200 \mathrm{~K}$ (crosses); $300 \mathrm{~K}$ (open circles). Also shown is the intensity obtained with $\mathrm{He}$ II at $300 \mathrm{~K}$ (open squares).

atoms distribute statistically on the surface upon adsorption at $300 \mathrm{~K}$ in agreement with the STM result.

\section{B. Ordered phases and transitions}

\section{Phases formed upon deposition at 100-200 K}

Two ordered phases are observed by LEED successively with increasing $\Theta_{\text {In }}$ upon deposition at $110 \mathrm{~K}$. A weak $c(3 \sqrt{2} \times \sqrt{2}) R 45^{\circ}$ LEED pattern appears at $\Theta_{\text {In }} \sim 0.25 \mathrm{ML}$, increases in intensity, and is most strongly observed at $\Theta_{\text {In }}$ $=0.65 \pm 0.03 \mathrm{ML}$ as shown in Fig. 7(a). At $\Theta_{\text {In }}$ above $\sim 0.75$, the fractional order spots become streaky along [110] and [100].

Figure 8(a) shows a high-resolution STM image taken on an In island on the surface with $\Theta_{\text {In }}=0.45 \mathrm{ML}$. In atoms are
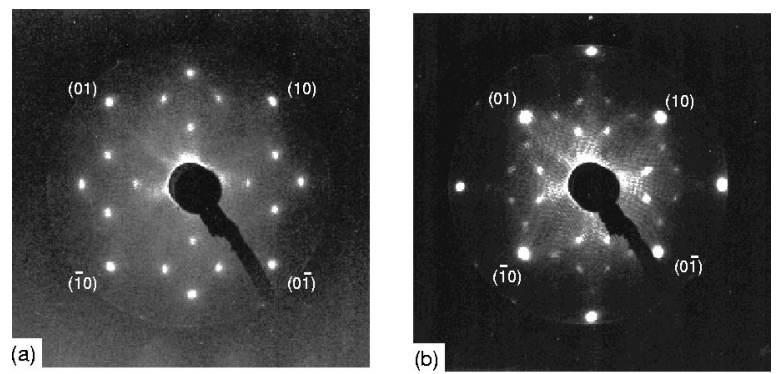

FIG. 7. LEED patterns observed at 110 and $200 \mathrm{~K}$ for In/ $\mathrm{Cu}(001)$ surface: (a) $c(3 \sqrt{2} \times \sqrt{2}) R 45^{\circ}$ (primary electron energy, $\left.E_{p}=103 \mathrm{eV}\right)$; (b) $(5 \sqrt{2} \times 5 \sqrt{2}) R 45^{\circ} \quad\left(E_{p}=114 \mathrm{eV}\right)$.

arranged along [010] with a spacing of $3.6 \AA\left(\sqrt{2} a_{0}\right)$. The STM image shows that there are two types of protrusions with different contrasts. The apparent height difference is $0.05 \AA$. The unit cell obtained by STM is $c(3 \sqrt{2}$ $\times \sqrt{2}) R 45^{\circ}$ in agreement with the LEED result, which is marked with the white lines in Fig. 8(a). A possible model structure deduced from the STM image is presented in Fig. $8(\mathrm{~b})$, in which the higher In atoms are placed on bridge sites while the lower ones on hollow sites. The In atoms constitute a 1D chainlike structure along [310] direction. The nearestneighbor distance is estimated to be $\sim 2.9 \AA$, considering the corrugation. This model suggests the ideal coverage of $\Theta_{\text {In }}=2 / 3 \mathrm{ML}$.

Upon further In deposition on the $c(3 \sqrt{2} \times \sqrt{2}) R 45^{\circ}$ surface, the LEED pattern changes to one shown in Fig. 7(b) at

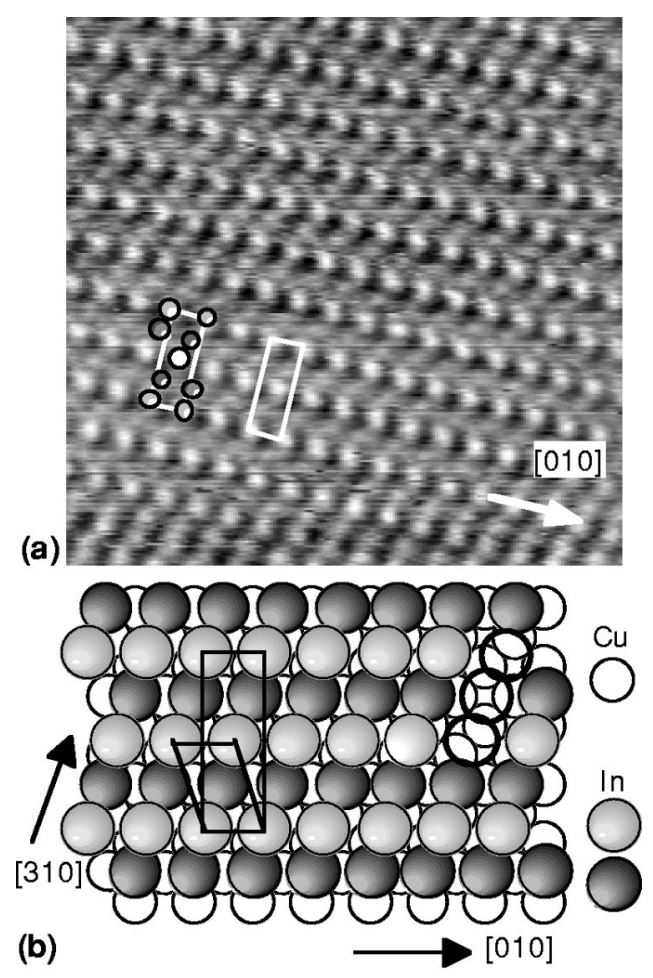

FIG. 8. (a) Atomically resolved STM image of the $c(3 \sqrt{2}$ $\times \sqrt{2}) R 45^{\circ}$ surface $\left(58 \times 58 \AA^{2}, V_{s}=-7 \mathrm{mV}, I_{t}=50 \mathrm{nA}\right)$. (b) $\mathrm{A}$ model proposed for the $c(3 \sqrt{2} \times \sqrt{2}) R 45^{\circ}$ surface. 


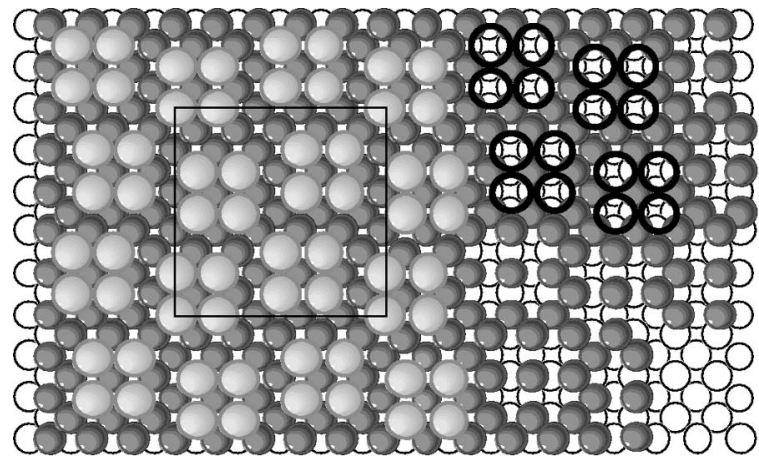

(a) Ocu substrate

In (2nd layer)

In (1st layer)

(01)

(b)

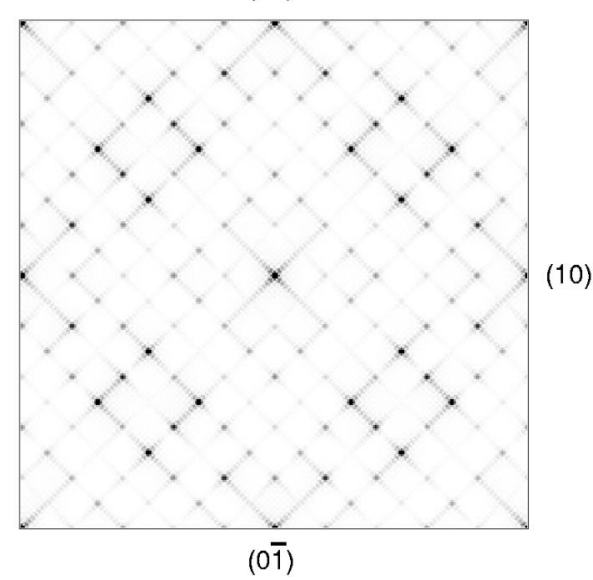

FIG. 9. (a) Possible configuration for the $(5 \sqrt{2} \times 5 \sqrt{2}) R 45^{\circ}$ surface. (b) Kinematically calculated LEED pattern for the structure shown in (a).

$\Theta_{\text {In }}=0.88$ ML. This pattern consists of (1/10)th order spots, and the spots positioned at $(h / 2 \pm 1 / 5, k / 2 \pm 1 / 5)$ with $h$ and $k$ odd and $(h \pm 1 / 5, k \pm 1 / 5)$ with $h$ and $k$ integers appear as intense spots. Though the LEED pattern could be indexed as

$$
\frac{1}{18}\left\{\begin{array}{ll}
25 & 15 \\
\overline{15} & 25
\end{array}\right\},
$$

or equivalently $(1.62 \times 1.62) R 31.0^{\circ}$, the arrangement of atoms according to this symmetry results in an unreasonable structure. This kind of superstructures has previously been reported in analogous systems, for example, $\mathrm{Pb} / \mathrm{Cu}(001)$ (Refs. 41,42) and $\mathrm{Bi} / \mathrm{Cu}(001)$ (Ref. 43). According to the proposal by Bocquet et al., ${ }^{42}$ the real space structure can be deduced by considering domain walls. The bright spots around $(1 / 21 / 2)$ indicate that the structure is composed of the local $c(2 \times 2)$ domains separated by domain wall network. ${ }^{42}$ Thus, this pattern can be interpreted as $(5 \sqrt{2}$ $\times 5 \sqrt{2}) R 45^{\circ}$. We propose a possible structural model for this surface as shown in Fig. 9(a). The model contains $c$ (2 $\times 2$ ) domains on the top layer which are in antiphase relationship with each other separated by domain boundaries between them. The second layer consists of In atoms of $\Theta_{\text {In }}$ $=0.68 \mathrm{ML}$, which is a density close to that of the $c(3 \sqrt{2}$ $\times \sqrt{2}) R 45^{\circ}$ structure model. A kinematically calculated
LEED pattern from the model structure is shown in Fig. 9(b), which is in fair agreement with the experimentally obtained LEED pattern shown in Fig. 7(b).

Additional In deposition onto the $(5 \sqrt{2} \times 5 \sqrt{2}) R 45^{\circ}$ surface increases the background intensity in LEED, and no further superstructure is observed.

Upon In deposition at $200 \mathrm{~K}$, the ordered structures observed by LEED were identical with those at $110 \mathrm{~K}$, though the LEED patterns appeared at slightly smaller deposition times.

\section{Phases formed upon deposition at $300 \mathrm{~K}$}

The LEED patterns observed upon deposition at $300 \mathrm{~K}$ are shown in Fig. 10. With increasing In coverage the LEED pattern changes from the clean surface $1 \times 1$ pattern to that shown in Fig. 10(a) around $\Theta_{\text {In }}=0.4$ ML. This LEED pattern becomes most intense around $\Theta_{\text {In }}=0.5 \mathrm{ML}\left(t_{\text {In }}\right.$ $=20 \mathrm{~min}$ ), coinciding with the first break point in the AES uptake curve. The pattern is characterized by sets of four $(1 / 18)$ th order spots in the vicinity of the half-order positions characteristic of a $p(2 \times 2)$ structure.

Figure 11(a) shows an atomically resolved STM image for the phase formed at $\Theta_{\mathrm{In}}=0.5 \mathrm{ML}$ at $300 \mathrm{~K}$, corresponding to the LEED pattern shown in Fig. 10(a). The surface consists of stripes of two types of structures. One of the domains consists of bright and dark protrusions arrayed in a distorted square lattice, and are separated by domains in which bright protrusions are arrayed in close-packed rows along the [110] and $[1 \overline{1} 0]$ directions. The average spacing between neighboring bright and dark protrusions in the distorted square domains is $\sim 2.6 \AA$, and hence these protrusions constitute a $c(2 \times 2)$-like structure. In the latter domain, dark protrusions can also be seen between rows of bright protrusions. The density of the protrusions is 0.5 ML each for bright and dark protrusions, suggesting that the bright and dark protrusions may correspond to topmost In and underlying $\mathrm{Cu}$ atoms, respectively. The contrast of the dark protrusions depends on the condition of STM tips. The unit cell of this structure is shown by a rectangle in Fig. 11(a), which is indexed as $(9 \sqrt{2} \times 2 \sqrt{2}) R 45^{\circ}$. The Fourier transform of the STM image for an area of $200 \times 200 \AA^{2}$ is shown in Fig. 11(c), which is qualitatively identical with the observed LEED pattern [Fig. 10(a)], except that the LEED pattern consists of contributions from two equivalent domains rotated by $90^{\circ}$ with each other. It is noted that the neighboring $c(2 \times 2)$-like domains are in antiphase relation, which makes the $(1 / 2,1 / 2)$ LEED spots missing.

At coverages beyond $\Theta_{\mathrm{In}}=0.6 \mathrm{ML}$, the LEED pattern changes to a $c(4 \times 4)$ pattern as shown in Fig. 10(c), which becomes most intense around $\Theta_{\mathrm{In}}=0.65 \mathrm{ML}$. Figure 11(c) shows an STM image for the $c(4 \times 4)$ surface. The density of protrusions is $5 / 8$, which is in good agreement with the In coverage.

With further increasing In coverage, the LEED pattern shown in Figs. 10(e) $-10(\mathrm{~g})$ appears, which is most intense around $\Theta_{\mathrm{In}}=1.0 \mathrm{ML} \quad\left(t_{\mathrm{In}}=40 \mathrm{~min}\right)$, coinciding with the second break in the AES uptake curve. This LEED pattern consists of strong half-order spots characteristic of a 

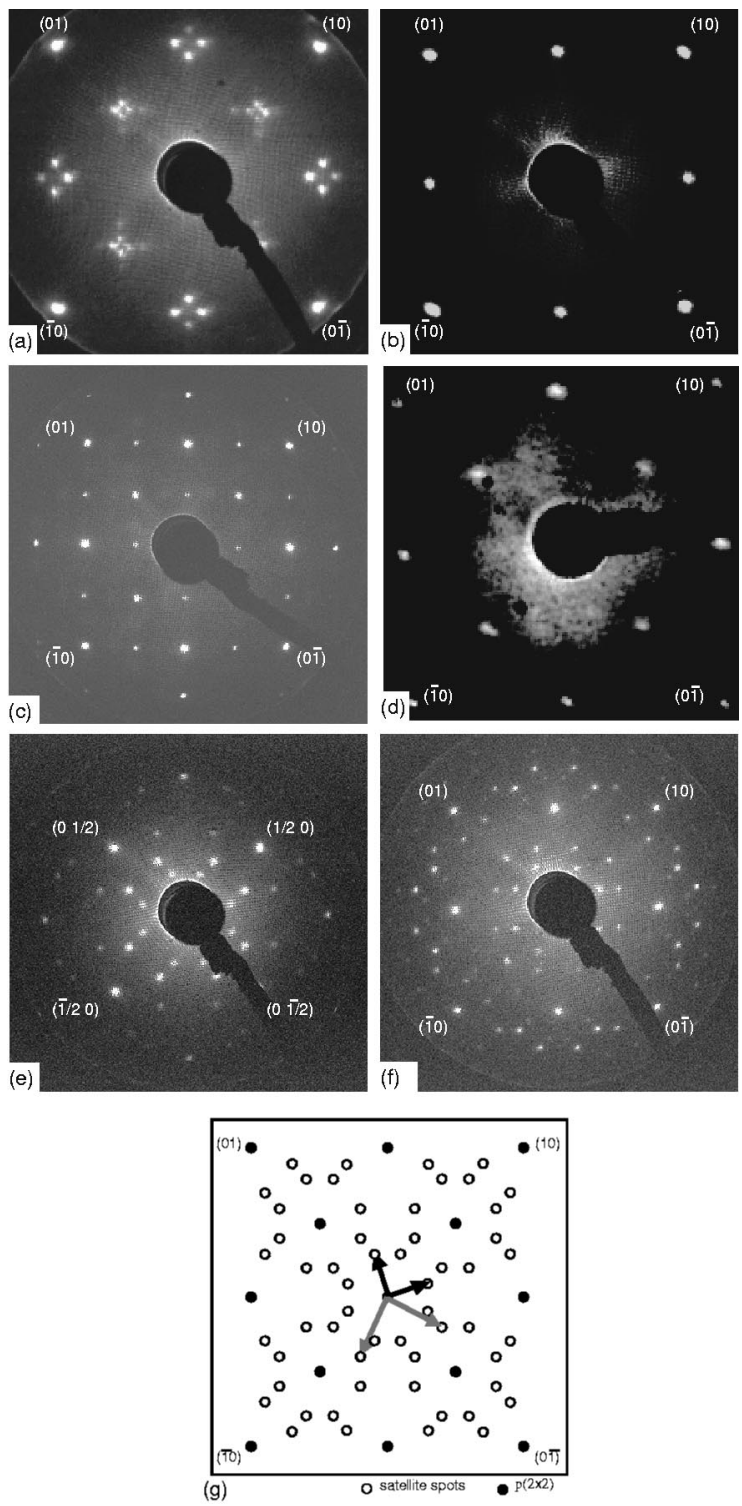

FIG. 10. LEED patterns for $\mathrm{In} / \mathrm{Cu}(001)$ surfaces upon In deposition at $300 \mathrm{~K}$ : (a) $(9 \sqrt{2} \times 2 \sqrt{2}) R 45^{\circ}\left(E_{p}=60 \mathrm{eV}\right)$; (b) $c(2$ $\times 2) \quad\left(E_{p}=60 \mathrm{eV}\right) ; \quad(\mathrm{c}) \quad c(4 \times 4)\left(E_{p}=114 \mathrm{eV}\right) ; \quad$ (d) $p(2$ $\times 2) \quad\left(E_{p}=90 \mathrm{eV}\right)$; (e) $\sqrt{20} \times \sqrt{20} R 64^{\circ}+p(2 \times 2) \quad\left(E_{p}=40 \mathrm{eV}\right)$; (f) the same as (e) but with $E_{p}=89 \mathrm{eV}$. (g) Schematic view of the $\sqrt{20} \times \sqrt{20} R 64^{\circ}+p(2 \times 2)$ pattern. Unit vectors are shown for $\sqrt{20} \times \sqrt{20} R 63.4^{\circ}$ by black arrows and for $\sqrt{10} \times \sqrt{10} R 18.4^{\circ}$ by gray arrows.

$p(2 \times 2)$ structure and numerous higher-order spots. These higher-order spots can be interpreted as due to the diffraction by $(\sqrt{10} \times \sqrt{10}) R 18.4^{\circ}$ or $(\sqrt{20} \times \sqrt{20}) R 63.4^{\circ}$ structure and the double diffraction by these structures and $p(2 \times 2)$ structure. While the intensity distribution of the spots may prefer the interpretation in terms of $(\sqrt{10} \times \sqrt{10}) R 18.4^{\circ}$, it cannot be strictly determined solely by kinematical consideration. Further In deposition beyond $\Theta_{\text {In }}=1.0 \mathrm{ML}$ makes the LEED spots disappear and the background intensity higher, indicating that the surface loses order.

Figure 11(e) shows an atomically resolved STM image of the In covered $\mathrm{Cu}(001)$ surface of $\Theta_{\text {In }}=1.0 \mathrm{ML}$ for which
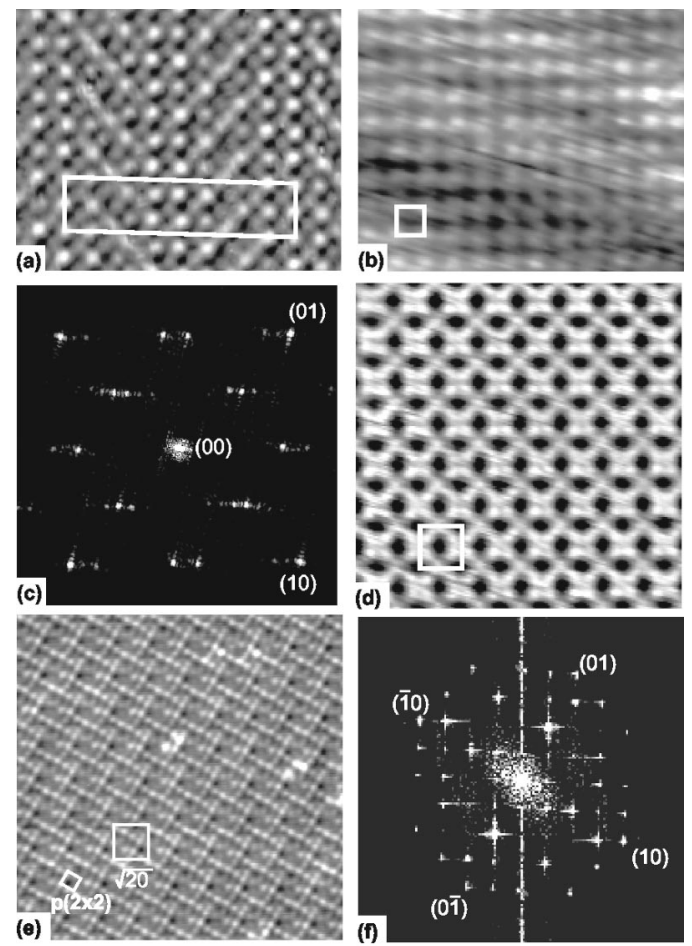

FIG. 11. (a) Atomically resolved STM images of $\mathrm{Cu}(001)-(9 \sqrt{2} \times 2 \sqrt{2}) R 45^{\circ}$-In. A unit cell is marked with white lines $\left(40 \times 40 \AA^{2}, V_{s}=-5 \mathrm{mV}, I_{t}=4 \mathrm{nA}\right)$. (b) STM image for the same surface as (a) but taken at $420 \mathrm{~K}$, showing $c(2 \times 2)$ structure $\left(40 \times 40 \AA^{2}, V_{s}=-20 \mathrm{mV}, I_{t}=4 \mathrm{nA}\right)$. (c) Fourier transform of the STM image of the $(9 \sqrt{2} \times 2 \sqrt{2}) R 45^{\circ}$ surface. (d) STM image for $c(4 \times 4)$ taken at $300 \mathrm{~K}\left(58 \times 58 \AA^{2}, V_{s}=50 \mathrm{mV}, I_{t}=10 \mathrm{nA}\right)$. (e) STM image for $(\sqrt{20} \times \sqrt{20}) R 63.4^{\circ}+p(2 \times 2)$ taken at $300 \mathrm{~K}$. Unit cells are shown for $p(2 \times 2)$ and $(\sqrt{20} \times \sqrt{20}) R 63.4^{\circ}(105$ $\times 105 \AA^{2}, V_{s}=-7 \mathrm{mV}, I_{t}=2 \mathrm{nA}$ ). (f) Fourier transform of the STM image of the $(\sqrt{20} \times \sqrt{20}) R 63.4^{\circ}+p(2 \times 2)$ surface.

the LEED pattern shown in Figs. 10(e) and 10(f) is most intensely observed. The image appears to be constituted by a square lattice as is marked by the small square, whose size and orientation agree with the $p(2 \times 2)$ symmetry. It is noted that the $p(2 \times 2)$ lattice is modulated by another periodicity as shown by the large square, which corresponds to the $(\sqrt{20} \times \sqrt{20}) R 63.4^{\circ}$ periodicity. Hence, we refer to this surface as $(\sqrt{20} \times \sqrt{20}) R 63.4^{\circ}+p(2 \times 2)$. The existence of the $(\sqrt{20} \times \sqrt{20}) R 63.4^{\circ}$ symmetry is well recognized by the Fourier transformation of the image as shown in Fig. 11(f), which is in good agreement with the LEED pattern from this surface.

\section{Stability of the low-temperature phases}

As shown above, the surface structures observed upon deposition at $110 \mathrm{~K}$ are different from those formed at 300 $\mathrm{K}$. Annealing the $c(3 \sqrt{2} \times \sqrt{2}) R 45^{\circ}$ surface formed at $110 \mathrm{~K}$ up to room temperature resulted in the change of the LEED pattern to $(9 \sqrt{2} \times 2 \sqrt{2}) R 45^{\circ}, c(4 \times 4)$, or $(\sqrt{20}$ $\times \sqrt{20}) R 63.4^{\circ}+p(2 \times 2)$ according to the In coverage. The transition starts from $220-240 \mathrm{~K}$ and is completed around $300 \mathrm{~K}$. As for the surface with the $(5 \sqrt{2} \times 5 \sqrt{2})$ LEED pat- 
tern, the LEED pattern changes to the $(\sqrt{20} \times \sqrt{20}) R 63.4^{\circ}$ $+p(2 \times 2)$ pattern around $240 \mathrm{~K}$. The transitions are irreversible: The low-temperature LEED patterns are not recovered after cooling down the surface to $110 \mathrm{~K}$. We therefore conclude that the low-temperature phases, $c(3 \sqrt{2}$ $\times \sqrt{2}) R 45^{\circ}$ and $(5 \sqrt{2} \times 5 \sqrt{2}) R 45^{\circ}$, are metastable.

\section{Reversible phase transitions}

When heating the $(9 \sqrt{2} \times 2 \sqrt{2}) R 45^{\circ}$ surface around 350 $\mathrm{K}$, the surface undergoes a phase transition to $c(2 \times 2)$, whose LEED pattern is shown in Fig. 10(b). STM observation also demonstrates this transition. ${ }^{2}$ We show an STM image for the surface of $\Theta_{\text {In }}=0.5 \mathrm{ML}$ taken at $420 \mathrm{~K}$ in Fig. 11(b), which shows clearly the $c(2 \times 2)$ structure. The changes in LEED and STM are fully reversible with respect to the temperature change. There is no change in the AES intensity during the phase transition, suggesting that there is no atom transfer between the overlayer and the substrate. Since the In coverage of this surface is $0.5 \mathrm{ML}$, the dark protrusions observed in the STM image of the $(9 \sqrt{2}$ $\times 2 \sqrt{2}) R 45^{\circ}$ surface are ascribed to electron density due to the substrate $\mathrm{Cu}$ atoms just beneath the In layer. The dark protrusions are not observed in the $c(2 \times 2)$ phase due probably to the reduced spatial resolution at elevated temperatures. The previous ARUPS study ${ }^{2}$ indicated that this phase transition is induced by the nesting of the 2D Fermi contour constituted by an In-induced surface resonance band.

The $c(4 \times 4)$ phase also exhibits a phase transition upon annealing. The LEED pattern shows that the surface undergoes a transition around $450 \mathrm{~K}$ to $p(2 \times 2)$ as shown in Fig. $10(\mathrm{~d})$. The $p(2 \times 2)$ phase is stable up to at least $550 \mathrm{~K}$. The AES result suggests that the In coverage does not change during the transition.

Heating the $(\sqrt{20} \times \sqrt{20}) R 63.4^{\circ}+p(2 \times 2)$ phase up to $\sim 450 \mathrm{~K}$ induces a phase transition to $p(2 \times 2)$. Annealing at $550 \mathrm{~K}$ for several minutes followed by cooling down to room temperature results in the $c(4 \times 4)$ LEED pattern. The AES intensities of $\mathrm{In}(\mathrm{Cu})$ also exhibit decrease (increase) to the value corresponding to the $c(4 \times 4)$ phase, suggesting the penetration into the substrate or the desorption of In atoms. It is noted that when the annealing is done quickly $(<1 \mathrm{~min}$ at $550 \mathrm{~K})$ the surface gets back to $(\sqrt{20} \times \sqrt{20}) R 63.4^{\circ}+p(2$ $\times 2)$ phase, while $(1 / 10)$ th order spots become weaker and the AES intensities exhibits noticeable changes, indicating that the penetration (desorption) rate is relatively low $(\sim 0.1 \mathrm{ML} / \mathrm{min})$ at $550 \mathrm{~K}$.

Upon cooling the phases formed at $300 \mathrm{~K}$, the LEED patterns observed at $300 \mathrm{~K}$ do not show any distinct phase transitions down to $110 \mathrm{~K}$. For the $c(4 \times 4)$ phase, the increase of the background at certain positions was observed at $110 \mathrm{~K}$, which implies the possibility of another phase transition below $110 \mathrm{~K}$.

\section{Evolution of In-derived surface electronic structure}

In this section we present the ARUPS results for the evolution of In-derived surface resonance band. In a previous publication, ${ }^{2}$ we reported that the In-induced surface reso-

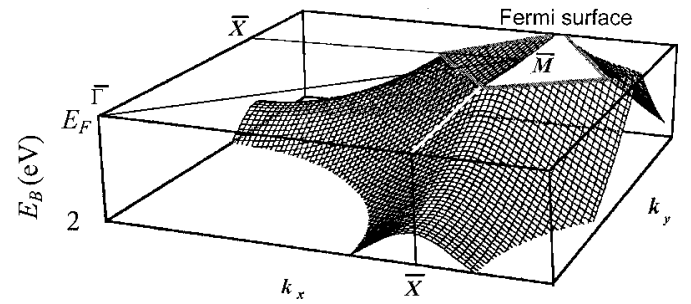

FIG. 12. Schematic drawing of the dispersion of the surface resonance band, $S_{1}$, for $\mathrm{Cu}(001)-c(2 \times 2)-\mathrm{In}$, which is a hightemperature phase at $\Theta_{\text {In }}=0.5 \mathrm{ML}$.

nance band $\left(S_{1}\right)$ gives rise to a squarelike Fermi surface around $\bar{M}$ in the high-temperature $c(2 \times 2)$ phase at $\Theta_{\text {In }}$ $=0.5 \mathrm{ML}$, and that the nesting of this Fermi surface drives the surface phase transition at $\sim 350 \mathrm{~K}$. The dispersion of the $S_{1}$ band was shown to mimic the edge of the projected bulk $4 s p$ band of $\mathrm{Cu}$. Figure 12 shows schematically the two-dimensional dispersion of the surface resonance band for the high-temperature $c(2 \times 2)$ phase at $\Theta_{\text {In }}=0.5$ ML. In the present work, we have studied the evolution of the $S_{1}$ resonance as a function of In coverage and surface temperature. It will be shown below that the $S_{1}$ resonance band appears to split off from the $\mathrm{Cu} 4 s p$ band upon the dealloying transition. Also shown is the photoemission result for the In $4 d$ core level.

\section{Valence-band structure at $300 \mathrm{~K}$}

The ARUPS spectra measured at an emission angle of $\theta_{e}=37.0^{\circ}$ in [110] direction, which corresponds to the $\bar{X}$ point for the binding energies of interest, are shown in Fig. 13(a). (The work function change in this coverage region is $0.3 \mathrm{eV}$, which corresponds to the change in $k$ of

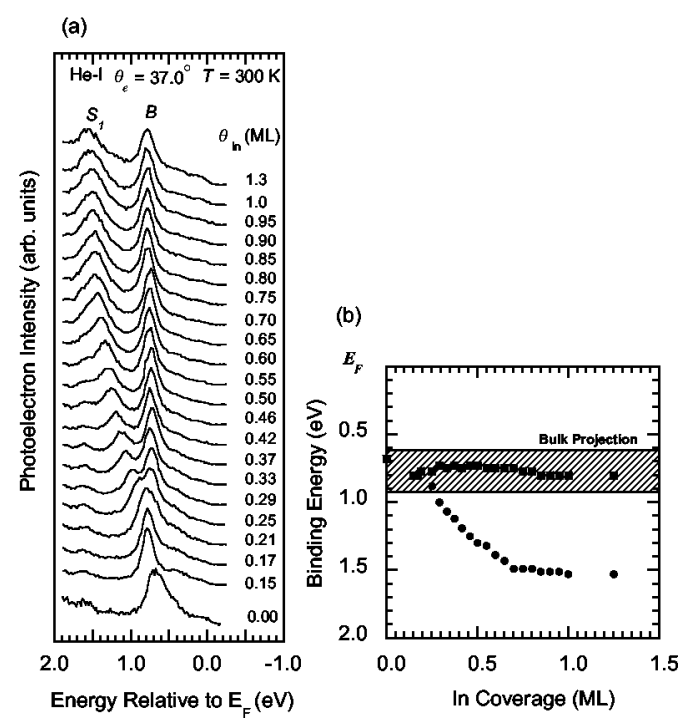

FIG. 13. (a) ARUPS spectra at $\bar{X}$ as a function of In coverage at $300 \mathrm{~K} . S_{1}$ denotes the In-induced surface resonance and $B$ the bulk $\mathrm{Cu} 4 s p$ band. (b) Binding energy for the $S_{1}$ (circle) and $\mathrm{Cu} 4 s p$ (square) bands at $\bar{X}$ as a function of In coverage. The hatched area indicates the projected bulk $\mathrm{Cu} 4 s p$ band at $\bar{X}$. 
$\sim 0.02 \AA^{-1}$.) In addition to the peak due to the bulk $\mathrm{Cu} 4 s p$ band, $B$, a peak induced by In adsorption is observed, $S_{1}$. Upon In deposition at $300 \mathrm{~K}$, the $S_{1}$ peak first emerges as a shoulder of $B$ around $0.25 \mathrm{ML}$, shifts downward in energy with increasing In coverage. The intensity of the $S_{1}$ peak grows as In coverage increases, reaches a maximum at $\sim 0.65 \mathrm{ML}$, and then it decreases significantly above $1 \mathrm{ML}$, which indicates that the $S_{1}$ peak is due to an electronic state localized at the $\mathrm{Cu}$-In interface. Note that the weak feature around $1.6 \mathrm{eV}$ in Fig. 13(a) is due to the excitation of the $\mathrm{Cu}$ $3 d$ band by $\mathrm{He}_{\beta}(h \nu=23.1 \mathrm{eV})$.

The $\mathrm{Cu}$ bulk state $B$ also shows a shift of $+0.15 \mathrm{eV}$ at small In coverages, and its line shape changes from an asymmetric one for the clean surface to a symmetric one for the In-covered surfaces. While the change in $k$-space sampling associated with the work-function change would result in the shift of $B$, it is estimated to be $<0.05 \mathrm{eV}$. The observed shift and line shape change are explained by a suppression of the indirect surface photoemission channel due to the removal of the dielectric mismatch between $\mathrm{Cu}$ and vacuum by In deposition. $^{44}$

The variation of the binding energy of $S_{1}$ is plotted against In coverage in Fig. 13(b). For the In coverage below $0.2 \mathrm{ML}$, the $S_{1}$ band is not clearly observed, but only the broadening of $B$ is discerned. Above $\Theta_{\text {In }}=0.25 \mathrm{ML}$, the $S_{1}$ band splits off from $B$ and grows in intensity. This coverage value corresponds to the dealloying transition from a surface alloy phase to separated phases of an In overlayer and the $\mathrm{Cu}$ substrate with an abrupt interface. With increasing In coverage, the $S_{1}$ peak shifts to higher binding energy and reaches the saturation value, $1.5 \mathrm{eV}$, at $\Theta_{\text {In }} \sim 0.65 \mathrm{ML}$. It is noted that a similar shift to higher binding energy upon initial In deposition is observed for the intrinsic surface state of $\mathrm{Cu}(001)$ at $\bar{M}$ as shown in Fig. 6 .

Figures 14(a) and 14(b) show the ARUPS spectra taken at different emission angles along [110] at $\Theta_{\text {In }}=0.25$ and 0.5 ML, respectively. In Fig. 14(d) the dispersion of the $S_{1}$ band along $\bar{\Delta}$ is plotted for different In coverages. At $\Theta_{\text {In }}$ $=0.25 \mathrm{ML}$, the $S_{1}$ band runs along the edge of the projected $\mathrm{Cu}$ bulk band. With increasing In coverage the whole band is shifted downward, splitting off from the bulk bands into the projected band gap for $\mathrm{Cu}(001)-1 \times 1$. Note, however, that the intrinsic band gap for the $\mathrm{Cu}(001)$ surface disappears for the In-covered surfaces due to the scattering by disordered In atoms at low coverages or to the Umklapp scattering in the superstructures formed at $\Theta_{\text {In }} \geqslant 0.5 \mathrm{ML}$. We therefore conclude that the $S_{1}$ band is due to an In-induced resonance state. The coverage-dependent behavior of $S_{1}$ and the resemblance of the dispersion with that of the $\mathrm{Cu}$ bulk band edge may suggest that the In-induced $S_{1}$ resonance state inherits a character of the $\mathrm{Cu} 4 \mathrm{sp}$ band.

Figure 14(c) shows the dispersion of the $S_{1}$ band for $\Theta_{\text {In }}=0.5 \mathrm{ML}$ at $400 \mathrm{~K}$, while the data in Fig. 14(b) are for $300 \mathrm{~K}$. At this coverage, the $\mathrm{In} / \mathrm{Cu}(001)$ surface undergoes a phase transition at $\sim 350 \mathrm{~K}$ from low temperature $(9 \sqrt{2}$ $\times 2 \sqrt{2}) R 45^{\circ}$ to high temperature $(\sqrt{2} \times \sqrt{2}) R 45^{\circ}[c(2$ $\times 2)$ ]. In Figs. 14(b) and 14(c), no significant differences are recognized between the two phases. In the previous study,
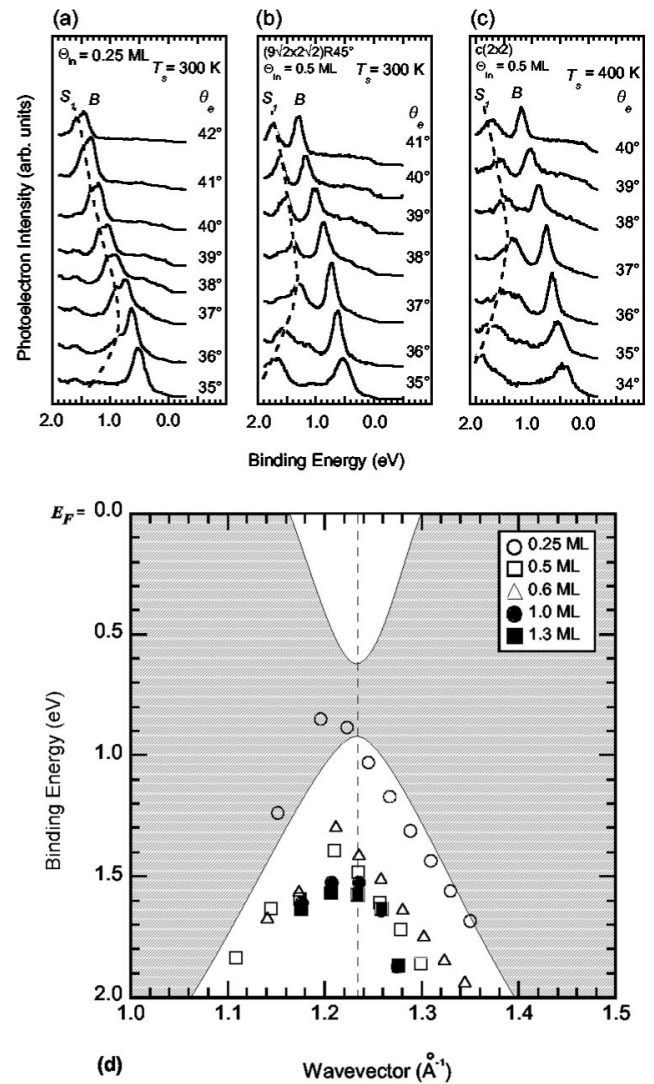

FIG. 14. (a) ARUPS spectra around $\bar{X}$ along [110] $(\bar{\Delta})$ for the surface with $\Theta_{\text {In }}=0.25 \mathrm{ML}$ at $300 \mathrm{~K}$. (b) The same as (a) but for the surface with $\Theta_{\text {In }}=0.5 \mathrm{ML}$ at $300 \mathrm{~K}$. (c) The same as (b) but measured at $400 \mathrm{~K}$. (d) Dispersion of the $S_{1}$ band with at different In coverages. The shaded area indicates the $\mathrm{Cu}$ bulk bands projected onto (001).

we showed that the surface resonance exhibits significant change upon the transition at $k$ points where the $S_{1}$ crosses the Fermi level in the high-temperature phase. ${ }^{2}$ The insensitivity of the $S_{1}$ resonance band along $\bar{\Delta}$, where the band does not approach the Fermi level, indicates that the change in electronic structure occurs only at very limited $k$ space close to the Fermi surface, which is consistent with the picture that the phase transition is due to a nonlocal electronic effect.

\section{In $4 d$ core level at $300 \mathrm{~K}$}

Changes in atomic and valence electronic structure may influence the binding energies and line shapes of the core levels of surface atoms. We measured high-resolution photoemission spectra of In $4 d$ level in different surface phases aiming, in particular, at possible core-level splitting in the CDW phase. In Figs. 15(a)-15(f), the In $4 d$ core level spectra taken at $300 \mathrm{~K}$ are shown for various In coverages. The feature of the spectrum at $\Theta_{\text {In }}=6 \mathrm{ML}$ is similar to the previous work for thick In films. ${ }^{45}$ In order to gain quantitative information, the spectra were fitted with a Doniach-Sunjić function. ${ }^{46,47}$ The spectra were fitted well with only a single component. There was some difference in the peak width between two spin-orbit-split lines, which was accounted for 


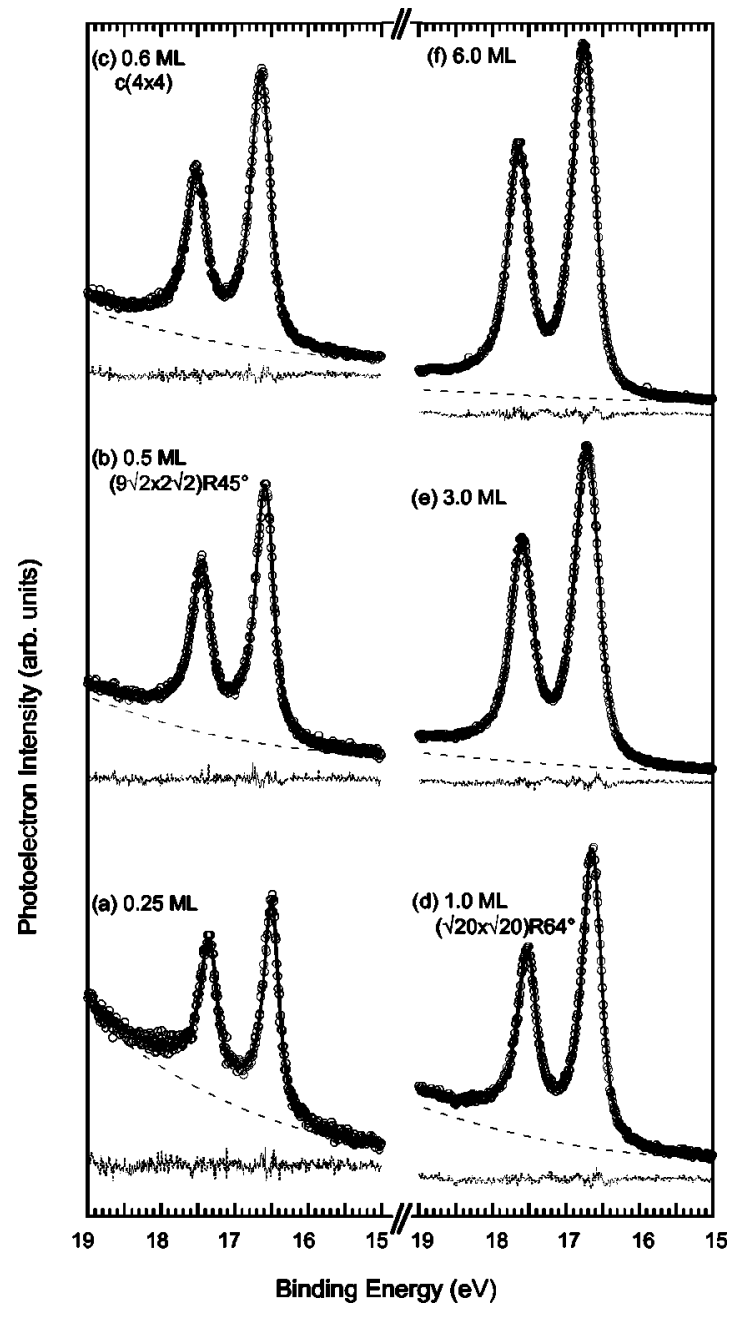

FIG. 15. In $4 d$ core level spectra taken at $300 \mathrm{~K}$ as a function of In coverage: (a) 0.25, (b) 0.50, (c) 0.6, (d) 1.0, (e) 3.0, (f) 6.0 ML. Filled circles represent raw data, solid lines are the results of the fitting by Doniach-Sunjić function. The dashed and thin solid lines show the background and the residuals, respectively.

by independent Lorentzian lifetime widths. The fitted lines and residuals are shown with thick and thin solid lines, respectively, in Fig. 15.

While Lorentzian lifetime width does not significantly change with increasing coverage, Gaussian width increases considerably (Fig. 16). At the smallest coverage studied ( $0.25 \mathrm{ML})$, the Gaussian width is $95 \mathrm{meV}$. Taking account of the spectrometer energy resolution of $60 \mathrm{meV}$, the phonon broadening is estimated to be $70 \mathrm{meV}$. The increase in the Gaussian width is mainly ascribed to the band formation which is induced by the In-In interaction at higher coverages. A theoretical calculation indicates that bulk In $4 d$ has a band width of a few hundred $\mathrm{meV}^{48}$ which is in fair agreement with the observed Gaussian width. Beyond the completion of the first monolayer at $0.65 \mathrm{ML}$, inhomogeneous broadening may also contribute to the increase of the Gaussian width. While the STM observation indicated that In grows in layerby-layer mode above $1 \mathrm{ML}$, coexistence of the In layer with different thickness may contribute to the Gaussian width.

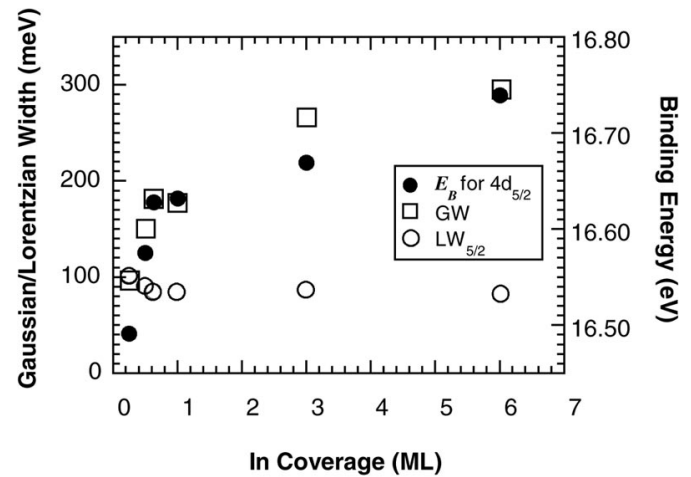

FIG. 16. Binding energy, Gaussian and Lorentzian widths of In $4 d_{5 / 2}$ as a function of coverage.

Depending on In coverage, the $4 d$ doublet shows shift from $16.49 \mathrm{eV}$ at $\Theta_{\text {In }}=0.25 \mathrm{ML}$ to $16.74 \mathrm{eV}$ at $\Theta_{\text {In }}$ $=6 \mathrm{ML}$ (Fig. 16). The latter value is in good agreement with that reported for a polycrystalline In film, $16.75 \mathrm{eV}^{45}$ which suggests that a 6-ML film already has bulk properties. The binding energy for submonolayer In is lower than that in bulk state. With increasing coverage, the binding energy is shifted quickly by $\sim 200 \mathrm{meV}$ up to $\sim 0.6 \mathrm{ML}$, where the slope gets gradual, and then approaches the bulk value asymptotically. Note that the first In monolayer is completed at $0.65 \mathrm{ML}$ and subsequent deposition results in the secondlayer growth.

Upon the growth at $300 \mathrm{~K}$, several long-range ordered phases are formed: $(9 \sqrt{2} \times 2 \sqrt{2}) R 45^{\circ}$ at $0.5 \mathrm{ML}, c(4 \times 4)$ at $0.65 \mathrm{ML}$, and $p(2 \times 2)+(\sqrt{10} \times \sqrt{10}) R 18.4^{\circ}$ at $1.0 \mathrm{ML}^{2} \mathrm{In}$ these structures, In atoms occupy several inequivalent sites. We, however, could not distinguish the different sites by core-level binding energies. We also checked whether the phase transition at $0.5 \mathrm{ML}$ between $(9 \sqrt{2} \times 2 \sqrt{2}) R 45^{\circ}$ and $c(2 \times 2)$ affect the shape of the core-level spectra. There was no significant changes except for the thermally induced phonon broadening in the high-temperature phase. Such insensitivity of the $4 d$ core level to the structure is in contrast to the case of In atoms on a Si surface, ${ }^{49}$ and is ascribed to the metallic nature of the system. Since the valence electrons with $s p$ character are well delocalized, the screening is effective enough to cancel out any changes due to the atomic environment.

\section{Valence-band structure at $110 \mathrm{~K}$}

Figure 17(a) shows the ARUPS spectra measured at the $\bar{X}$ point for $\mathrm{Cu}(001)$ deposited with In at $110 \mathrm{~K}$. In-derived surface state, denoted as $S_{1}^{\prime}$, is observed at almost the same energy region as the $S_{1}$ band was observed at $300 \mathrm{~K}$. The coverage-dependent shift of $S_{1}^{\prime}$, however, is different from that of $S_{1}$. (The nondispersing peak at $1.6 \mathrm{eV}$ is due to $\mathrm{Cu} 3 d$ excited by $\mathrm{He}_{\beta}$ emission.) With increasing In coverage, the surface state first emerges at $1.15 \mathrm{eV}$ at $\Theta_{\text {In }}=0.38 \mathrm{ML}$, grows in intensity shifting gradually to $1.20 \mathrm{eV}$ at $\Theta_{\text {In }}$ $=0.75 \mathrm{ML}$, and shifts abruptly to $\sim 1.6 \mathrm{eV}$. The intensity decreases above 1.0 ML, which indicates that the $S_{1}^{\prime}$ band is also due to an interfacial electronic state. 

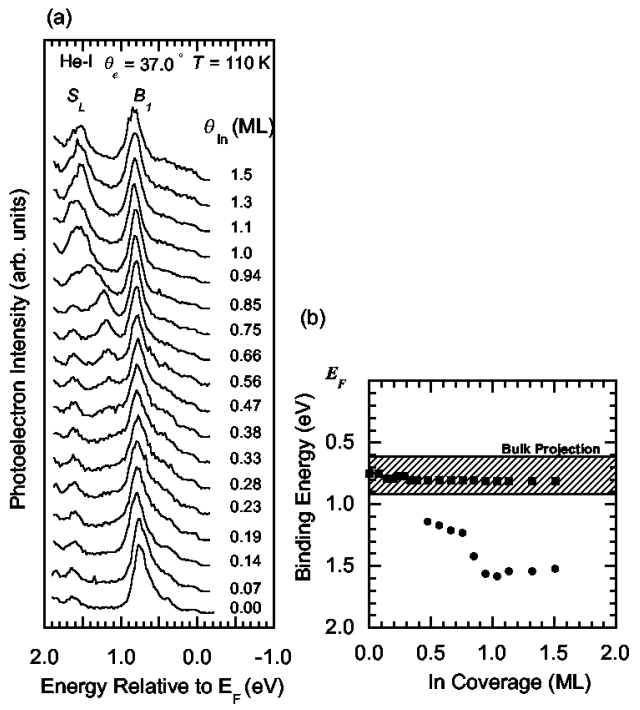

FIG. 17. (a) ARUPS spectra at $\bar{X}$ as a function of In coverage for the surface grown at $110 \mathrm{~K}$. (b) Binding energy for the $S_{1}^{\prime}$ (circle) and $\mathrm{Cu} 4 s p$ (square) bands at $\bar{X}$ as a function of In coverage. The hatched area indicates the projected bulk $\mathrm{Cu} 4 s p$ band at $\bar{X}$.

In contrast to the growth at $300 \mathrm{~K}$, where mixed alloy layer is initially formed and then switched to the overlayergrowth mode, In atoms deposited at $110 \mathrm{~K}$ are initially adsorbed as isolated adatoms and then gradually forms $2 \mathrm{D}$ islands, as indicated by the appearance of a $c(3 \sqrt{2}$ $\times \sqrt{2}) R 45^{\circ}$ LEED pattern at $\sim 0.3 \mathrm{ML}$. Hence the appearance of the $S_{1}^{\prime}$ resonance is in coincidence with the formation of $2 \mathrm{D}$ islands. The $c(3 \sqrt{2} \times \sqrt{2}) R 45^{\circ}$ layer is completed at $0.65 \mathrm{ML}$, beyond which the second layer starts to grow, which is also in coincidence with the sudden shift of the $S_{1}^{\prime}$ peak from 1.2 to $1.6 \mathrm{eV}$. We therefore conclude that the stepwise shift of the $S_{1}^{\prime}$ band is due to the 2D island growth of the first and the second In layers. The plateaus found in the binding energy of $S_{1}^{\prime}$ as a function of In coverage correspond to the interfacial states in 2D islands in the first and the second layer.

The ARUPS spectra measured along $\bar{\Delta}$ near the $\bar{X}$ point for $\Theta_{\text {In }}=0.8$ and 1.2 ML are shown in Figs. 18(a) and 18(b), respectively. As compared with the dispersion of the $S_{1}$ band for In overlayers formed at $300 \mathrm{~K}$, the $S_{1}^{\prime}$ band show little dispersion along $\bar{\Delta}$ and the intensity is strongly reduced for $k$ away from $\bar{X}$. The dispersion plot in Fig. 18(c) shows that the $S_{1}^{\prime}$ band has a flat dispersion irrespective of the In coverage and are merged into the projected bulk band upon getting away from $\bar{X}$.

\section{DISCUSSION}

\section{A. Coverage calibration}

Before discussing the phase diagram and the growth mechanism, we give a brief account of the coverage calibration we have done in this work. The most reliable experimental facts to establish In coverage have been obtained by
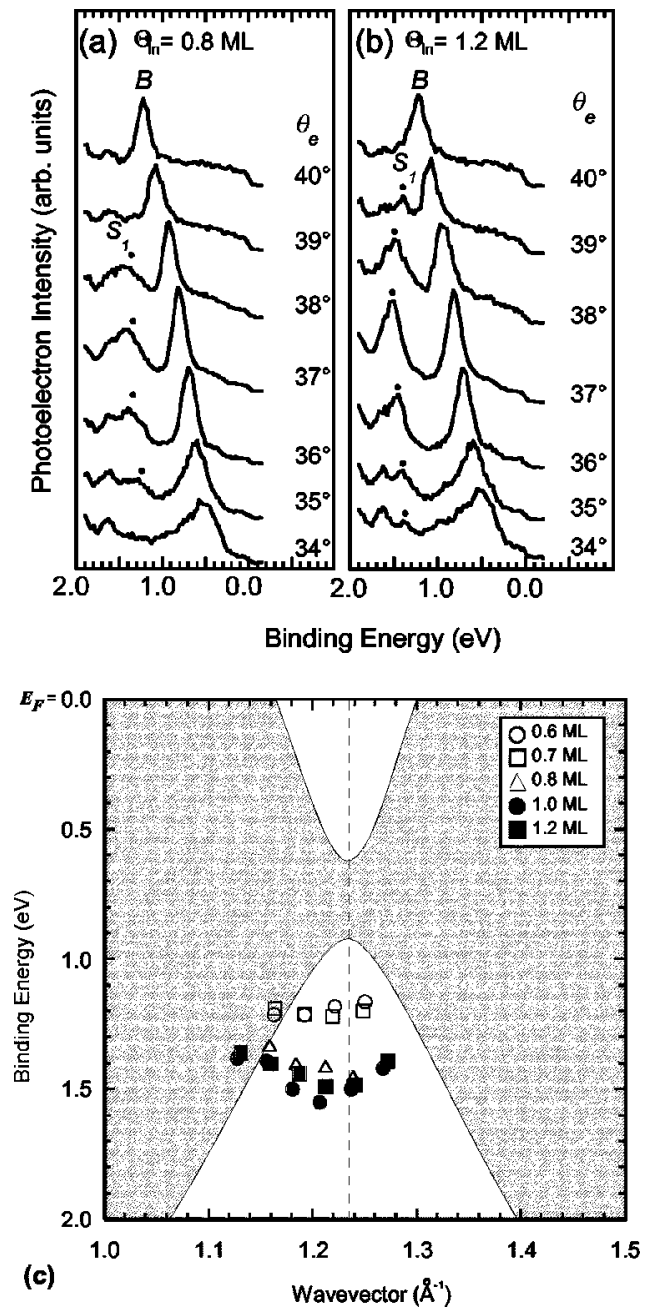

FIG. 18. An In-derived surface resonance band near $\bar{X}$ for (a) spectra for $\Theta_{\text {In }}=0.8 \mathrm{ML}$, and (b) spectra for $\Theta_{\text {In }}=1.2 \mathrm{ML}$. (c) Dispersion of the $S_{1}^{\prime}$ band at different In coverages. The shaded area indicates the $\mathrm{Cu}$ bulk bands projected onto (001).

directly counting the In atoms at initial stages of the growth. Based on the STM images taken at $t_{\text {In }}$ from 6 to $14 \mathrm{~min}$, the uptake rate of $0.025 \pm 0.001 \mathrm{ML} / \mathrm{min}$ has been deduced. The attenuation curve of $\mathrm{Cu}$ AES intensity supports this calibration.

While the above uptake rate was obtained for deposition at $300 \mathrm{~K}$, there are several facts indicating that the rate is not affected by the surface temperature during deposition.

(1) The Cu AES curves measured at 110 and $300 \mathrm{~K}$ exhibit similar variation as a function of $t_{\text {In }}$ except that the breaks are dulled at $110 \mathrm{~K}$.

(2) Upon deposition at or below $200 \mathrm{~K}$, where no surface alloy is formed, the $c(3 \sqrt{2} \times \sqrt{2}) R 45^{\circ}$ overlayer is obtained at $t_{\mathrm{In}}=26 \pm 1 \mathrm{~min}$. The model obtained from the STM image indicates that this structure corresponds to $\Theta_{\text {In }}=2 / 3 \mathrm{ML}$.

(3) Annealing the well-developed $c(3 \sqrt{2} \times \sqrt{2}) R 45^{\circ}\left(t_{\text {In }}\right.$ $=26 \mathrm{~min}$ ) surface up to $300 \mathrm{~K}$ resulted in the transformation to the well-developed $c(4 \times 4)$ structure, which is obtained by deposition at $300 \mathrm{~K}$ also for $t_{\mathrm{In}}=26 \mathrm{~min}$.

Thus, the coverage for any surface can be determined by the deposition time. Note that the coverage corresponding to 


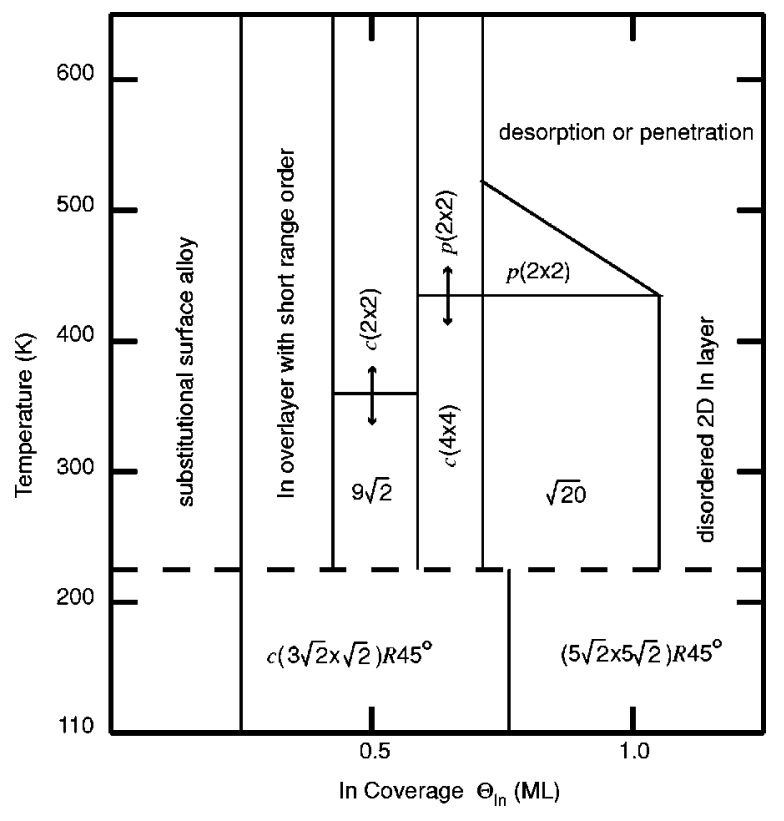

FIG. 19. Phase diagram for the $\mathrm{In} / \mathrm{Cu}(001)$ surface. " $9 \sqrt{2}$ ", and " $\sqrt{20}$ " denote the $(9 \sqrt{2} \times 2 \sqrt{2}) R 45^{\circ}$ and $(\sqrt{20} \times \sqrt{20}) R 63.4^{\circ}$ $+p(2 \times 2)$ phases, respectively. The $c(3 \sqrt{2} \times \sqrt{2}) R 45^{\circ}$ and $(5 \sqrt{2}$ $\times 5 \sqrt{2}) R 45^{\circ}$ phases are metastable and can be achieved only by deposition below $200 \mathrm{~K}$. The broken line indicates irreversible phase transitions, while the solid lines with arrows at both ends indicate reversible transitions.

the $(9 \sqrt{2} \times 2 \sqrt{2}) R 45^{\circ}$ phase was assumed to be $1.0 \mathrm{ML}$ in the previous paper, ${ }^{2}$ which was due to the lack of STM data for the initial growth.

\section{B. Phase diagram}

In Fig. 19, we summarize the phases observed for In/ $\mathrm{Cu}(001)$ in a $T-\Theta_{\text {In }}$ diagram for different deposition temperatures.

At $110-200 \mathrm{~K}$ In grows as overlayers and shows two ordered structures. Islands of the $c(3 \sqrt{2} \times \sqrt{2}) R 45^{\circ}$ structure are formed above $\Theta_{\text {In }}=0.25 \mathrm{ML}$. This phase is completed at $\Theta_{\text {In }}=0.67 \mathrm{ML}$. The $c(3 \sqrt{2} \times \sqrt{2}) R 45^{\circ}$ LEED pattern becomes streaky above $\Theta_{\text {In }}=0.75 \mathrm{ML}$ and then changes to the $(5 \sqrt{2} \times 5 \sqrt{2}) R 45^{\circ}$ pattern at $\Theta_{\text {In }}=0.88$ ML. The STM observation at $200 \mathrm{~K}$ shows that the exchange process between In and surface $\mathrm{Cu}$ atoms are hindered and that the 2D islands grow on top of the $\mathrm{Cu}(001)$ surface. Due to the low diffusion barrier of In on the $\mathrm{Cu}(001)$ surface, the growth occurs by step-flow growth. The $c(3 \sqrt{2} \times \sqrt{2}) R 45^{\circ}$ structure has an In density larger by $\sim 8 \%$ than that of the $\operatorname{In}(001)$ surface. The wide-scan STM results suggest that the transformation from the $c(3 \sqrt{2} \times \sqrt{2}) R 45^{\circ}$ structure to the $(5 \sqrt{2} \times 5 \sqrt{2}) R 45^{\circ}$ structure is associated with the formation of second In layer. These phases are metastable and transform irreversibly to the stable phases below $300 \mathrm{~K}$.

At $300 \mathrm{~K} \mathrm{In}$ is incorporated into the topmost layer of the $\mathrm{Cu}(001)$ substrate and forms surface alloy. At coverages $\Theta_{\text {In }} \sim 0.25 \mathrm{ML}$, the short-range order among incorporated In atoms begins to develop. The dealloying transition occurs around $\Theta_{\text {In }} \sim 0.35 \mathrm{ML}$ and the short-range order further develops to yield a long-range order, $(9 \sqrt{2} \times 2 \sqrt{2}) R 45^{\circ}$, at $\Theta_{\text {In }}=0.5 \mathrm{ML}$. We previously showed that the Fermi surface nesting in the high-temperature $c(2 \times 2)$ phase is the origin of the stabilization of the $(9 \sqrt{2} \times 2 \sqrt{2}) R 45^{\circ}$ structure. $^{2}$ The structural similarity implies that the short-range-order phase at $\Theta_{\text {In }} \sim 0.35-0.5 \mathrm{ML}$ also has the electronic origin. This is supported by the ARUPS experiment ${ }^{50}$ which shows that the electronic band structure of the short-range-order phase is similar to that of $(9 \sqrt{2} \times 2 \sqrt{2}) R 45^{\circ}$. Further increase of the In coverage gives rise to the $c(4 \times 4)$ structure and subsequently to the $(\sqrt{20} \times \sqrt{20}) R 63.4^{\circ}+p(2 \times 2)$ structure, and finally makes the surface disordered.

\section{Alloying at low In coverages}

Initial deposition of In on $\mathrm{Cu}(001)$ was previously studied by means of several experimental techniques. In order to compare our result with the previous ones, we here summarize briefly the previous assignments of the In occupation sites and the onset temperature of In incorporation. Perturbed $\gamma-\gamma$ angular correlation (PAC) method which uses $10^{-4} \mathrm{ML}$ radioactive ${ }^{111}$ In as a nuclear probe is sensitive to the configuration of the probe atoms, giving the information about the occupied sites. Klas et al. ${ }^{51}$ used this technique and suggested that, after annealing the surface with $\Theta_{\text {In }}$ $\sim 10^{-4}$ ML above $400 \mathrm{~K}$, all In atoms occupied the terrace substitutional site. In the subsequent publication from the same group, ${ }^{52}$ it was mentioned that they observed In adatoms on top of the $\mathrm{Cu}$ terraces at low temperatures. Breeman and Boerma ${ }^{26}$ performed a low-energy ion scattering (LEIS) experiment to study the sites and mobility of In atoms of $\sim 10^{-2}$ ML on a stepped $\mathrm{Cu}(17,1,1)$ surface which has steps every eight-atom length terrace. It was suggested that In atoms were adsorbed as isolated adatoms on terraces below $\sim 80 \mathrm{~K}$, above which In atoms became mobile and were adsorbed mainly at step edges and formed chains along the step below $\sim 150 \mathrm{~K}$. According to their analysis, the incorporation of In atoms into terrace starts at 80-90 K and In atoms embedded in terrace are the majority species above $150 \mathrm{~K}$. Recently, Roşu et al. ${ }^{53}$ studied the temperaturedependent behavior of In atoms on $\mathrm{Cu}(001)$ by PAC. They showed that the exchange process between In adatoms and substrate $\mathrm{Cu}$ atoms starts at temperatures between 180 and $300 \mathrm{~K}$, depending on the step density. There is thus a discrepancy about the transition temperature from the overlayer growth to the surface alloy formation.

The present STM result is in qualitative agreement with these works as to the occupation sites at low coverages $(<0.1 \mathrm{ML})$ : In atoms are adsorbed on terrace at low temperatures while they are incorporated into the topmost substrate layer to form surface alloy at high temperatures. As to the onset temperature of the exchange process, our results are in good agreement with that of Roşu et al. ${ }^{53}$ The STM images show that upon deposition at $200 \mathrm{~K}$ the In atoms form islands on terraces or at steps, and only a very small amount of In is incorporated into the terraces. Atomically resolved STM images show that only a few per cent of In atoms are embedded in terraces at $200 \mathrm{~K}$. The discrepancy between this 
and the previous suggestion based on LEIS result may be due to the formation of $2 \mathrm{D}$ islands at $200 \mathrm{~K}$, since In atoms in $2 \mathrm{D}$ islands cannot be distinguished by LEIS from those incorporated in terraces. ${ }^{26}$

The ARUPS study of the surface state gives us much information about the In growth. The photoemission intensity of surface state decreases linearly with increasing In coverage upon deposition at $300 \mathrm{~K}$, and finally disappears at $t_{\text {In }}$ $=6 \min \left(\Theta_{\text {In }}=0.15 \mathrm{ML}\right)$, which indicates the lateral extent of $\mathrm{Cu}$ substrate required to form the Tamm surface state. Deposition at $200 \mathrm{~K}$ resulted in the 2D island formation. The 2D islands quench completely the surface state for the covered area but exert little influence on bare terraces. Upon deposition at $110 \mathrm{~K}$, the decrease of the surface state intensity with almost the same rate as that at $300 \mathrm{~K}$ was observed up to $t_{\mathrm{In}} \sim 5 \mathrm{~min}\left(\Theta_{\mathrm{In}} \sim 0.13 \mathrm{ML}\right)$, beyond which the rate of the reduction of the surface state becomes moderate due to the formation of small 2D islands. There are some differences in the behavior of the surface state upon deposition at 110 and $300 \mathrm{~K}$. The reduction of intensity with respect to In deposition time at $300 \mathrm{~K}$ is $\sim 25 \%$ larger than that at $110 \mathrm{~K}$ in the initial deposition regime $\left(t_{\mathrm{In}}<5 \mathrm{~min}\right)$. The peak shift at $t_{\text {In }}=5$ min at $300 \mathrm{~K}$ is $\sim 80 \mathrm{meV}$, while the shift at the same deposition time at $110 \mathrm{~K}$ is only $30 \mathrm{meV}$. The stronger effect at $300 \mathrm{~K}$ indicates that the surface state is effectively influenced when the surface alloy is formed. This may be due to the fact that the surface state originates from the inplane $d_{x^{2}-y^{2}}$ states. $^{21}$

The formation of substitutional surface alloys at room temperature has frequently been observed in many metal/ $\mathrm{Cu}(001)$ systems. In the case of $\mathrm{Pb}$ whose radius is $37 \%$ larger than that of $\mathrm{Cu}, \mathrm{Pb}$ atoms are embedded in terraces up to a coverage of $0.375 \mathrm{ML}$, and the ordered $c(4 \times 4)$ surface alloy is formed. ${ }^{10} 0.5 \mathrm{ML}$ of $\mathrm{Pd},{ }^{11} \mathrm{Au},{ }^{7}$ and $\mathrm{Mn}$ (Ref. 54) atoms deposited on $\mathrm{Cu}(001)$ are known to form $c(2 \times 2)$ surface alloys. In the present case, however, substitutional surface alloy does not show any long-range order.

\section{Dealloying}

Substitutional surface alloys which maintain the $1 \times 1$ average periodicity of atoms sometimes show instability upon increase of the density of substituted atoms. In the $\mathrm{Au} /$ $\mathrm{Cu}(001)$ system, the surface with 0.5 -ML $\mathrm{Au}$ gives rise to misfits to relax the stress induced by the large size mismatch between $\mathrm{Au}$ and $\mathrm{Cu}^{7}$ In some cases the transformation from $1 \times 1$ average lattice is observed. In the $\mathrm{Au} / \mathrm{Ni}(110)$ system, a substitutional surface alloy is formed up to an Au coverage of 0.4 ML, in which Au atoms are confined in the $1 \times 1$ lattice sites randomly. Above 0.4 ML, however, a zigzag chain structure appears, in which $\mathrm{Au}$ forms dimer-trimer chains leaving vacancies. ${ }^{8}$

In the present work, we observed the substitutional surface alloy with $\mathrm{In}$ and $\mathrm{Cu}$ atoms occupying $1 \times 1$ lattice sites randomly up to $\Theta_{\text {In }}=0.25 \mathrm{ML}$. On the surface for $\Theta_{\text {In }}$ $=0.35 \mathrm{ML}, \mathrm{Cu}$ atoms were never imaged irrespective of the tunneling condition, and In form chain structures which are inconsistent with the $1 \times 1$ lattice. The work-function data also show a significant change above $\Theta_{\text {In }}=0.25 \mathrm{ML}$, which synchronizes with the disappearance of $\mathrm{Cu}$ atoms in the STM images. After the plateau for $\Theta_{\text {In }}=0-0.25 \mathrm{ML}$, the work function starts to decrease at a rate comparable to those observed for the adsorption on terraces at 110 and $200 \mathrm{~K}$. Based on these experimental facts, we suggest that the dealloying transition occurs around $\Theta_{\text {In }}=0.25-0.35 \mathrm{ML}$.

The stability of In atoms at various sites at the $\mathrm{Cu}(001)$ surface was studied by semiempirical methods. ${ }^{55,56}$ An In atom in a terrace substitutional site was found to be most stable, which is in agreement with the observation of interrace In atoms by STM, LEIS, and PAC. The total energy of pairs of embedded In atoms was also calculated, ${ }^{56}$ which showed that two embedded In atoms in next-nearestneighbor sites are destabilized as compared with the isolated ones, in agreement with the notion that In is oversized by $\sim 30 \%$ than $\mathrm{Cu}$. We suggest that the dealloying transition is driven by the destabilization of the substitutional surface alloy at high In concentrations.

Similar behavior of oversized adatoms of an $s p$ metal on $\mathrm{Cu}(001)$ has been reported for $\mathrm{Bi} / \mathrm{Cu}(001) .{ }^{57}$ Using surface $\mathrm{x}$-ray diffraction, it was concluded that $\mathrm{Bi}$ atoms substitute surface $\mathrm{Cu}$ atoms below about $0.35 \mathrm{ML}$ and that above this coverage the fractional $\mathrm{Cu}$ occupancy of the surface layer decreases rapidly with increasing $\mathrm{Bi}$ coverage.

The dynamics of the dealloying transition is of interest. Several possible scenarios can be supposed: (1) $\mathrm{Cu}$ atoms are pushed out onto the terrace when the local coverage of embedded $\mathrm{In}$ atoms exceeds a critical value, and these $\mathrm{Cu}$ atoms diffuse on the surface to attach to steps; (2) In atoms are pushed out onto the terrace and the resulting vacancies quickly diffuse to be filled with $\mathrm{Cu}$ atoms at steps in a way similar to that proposed recently. ${ }^{23}$

\section{E. Evolution of surface resonance and its relevance to the geometric structure}

We first discuss the mechanism of the evolution of the surface resonance bands. The coverage dependent measurements at $\bar{X}$ [Figs. 13(a) and 17(a)] imply that the influence of In adsorption onto the $\mathrm{Cu} 4 s p$ band gives rise to the surface bands, $S_{1}$ and $S_{1}^{\prime}$, split off from the bulk $\mathrm{Cu}$ band into the projected band gap. This leads to a picture in which the surface resonance has its origin in the $4 s p$ band in surface $\mathrm{Cu}$ layers but is modified due to the effect of In layers. For atoms at the surface, the bonds are lost due to the existence of the vacuum. This lack of bonding leads to the excess charge confined at the surface, resulting in the modification of the potential near the surface. It is known that such surface potential due to excess surface charge gives rise to the $\bar{M}$ surface state on $\mathrm{Cu}(001)$, which is due to the $d_{x^{2}-y^{2}}$ band pushed up into the projected band gap by the surface potential. ${ }^{19}$ When the $\mathrm{Cu}$ atoms at the surface make metallic bonds with In atoms, the confined electrons near the $\mathrm{Cu}$ atoms are spread over the interface, which lowers the surface potential. This view is supported by the downward shift of the $\bar{M}$ surface state upon In adsorption (Fig. 6). In a similar way, the lowering of the surface potential can also lead to the formation of interfacial state which is split off from the $\mathrm{Cu}$ $4 s p$ band. As shown in Fig. 20, there is a projected band gap 

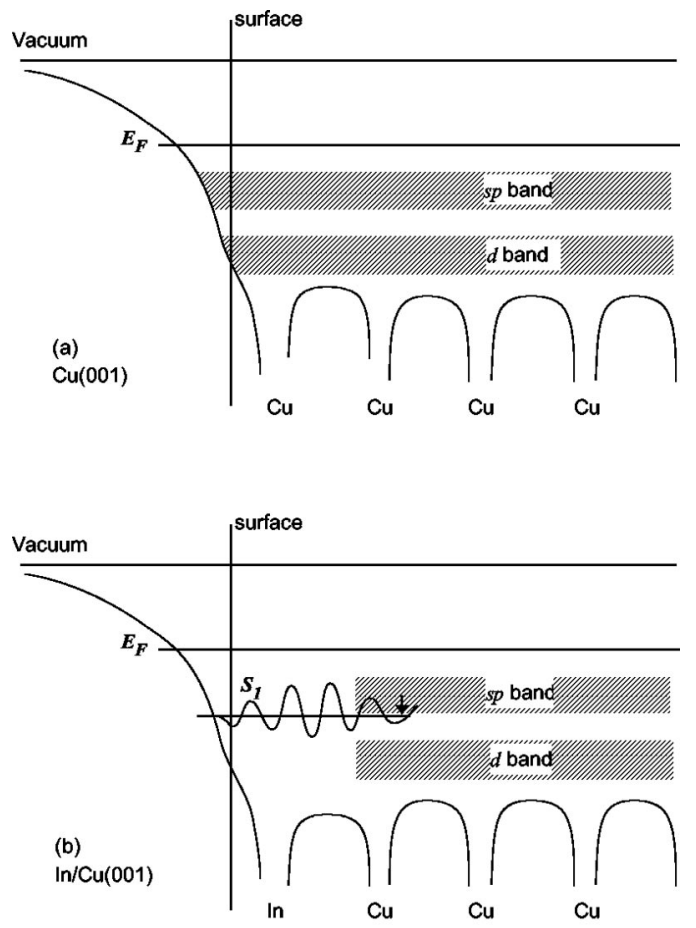

FIG. 20. Schematic representation of potential and energy bands at the surface of (a) $\mathrm{Cu}(001)$ and (b) $\mathrm{In} / \mathrm{Cu}(001)$ around the $\bar{X}$ symmetry point. The formation of an In-derived interface state is indicated in (b).

between the $s p$ and $d$ bands of $\mathrm{Cu}$ at $\bar{X}$. The interfacial state therefore should have a large amplitude at the In-Cu interface, though it is not a true interface state since the band gap actually disappears by coupling associated with reciprocal lattice vectors in the superstructures.

Alternatively, the evolution of the surface resonance may well be described in the language of metallic bonding between $\mathrm{Cu}$ and $\mathrm{In}$. The hybridization between $\mathrm{Cu} 4 s p$ and $\mathrm{In}$ $5 s p$ states should result in the formation of interfacial states with bonding and antibonding characters. The shift to higher binding energies with increasing In coverage in the submonolayer region is ascribed to the enhanced bonding when the number of $\mathrm{In}$ atoms in contact with $\mathrm{Cu}$ is larger. The fact that the $S_{1}$ surface resonance exhibits an extensive dispersion even at low coverages may suggest that the interfacial states are not of localized, covalent bonding nature but much more of laterally delocalized, metallic one. These two pictures do not necessarily contradict each other but may serve complementary descriptions of the interfacial electronic state. Firstprinciples electronic structure calculations would reveal the precise character of the $S_{1}$ surface resonance.

The observed variation of the $S_{1}$ surface resonance as a function of coverage is quite moderate at room temperature: After it splits off from the bulk $4 s p$ band, only a gradual shift to higher binding energies is observed, which is then saturated at $\Theta_{\text {In }} \sim 0.65 \mathrm{ML}$. On the other hand, concomitant with the shift of the $S_{1}$ resonance, the surface In atoms exhibit remarkable structural changes at room temperature as a function of coverage. Since the atomic arrangement should in principle be determined by electronic energies, the inter- play between geometrical and electronic structure is of interest. For the $(9 \sqrt{2} \times 2 \sqrt{2}) R 45^{\circ}$ structure formed at $\Theta_{\text {In }}$ $=0.5 \mathrm{ML}$, we have previously shown that the " $9 \sqrt{2}$ " periodicity is determined by the Fermi surface nesting. ${ }^{2}$ While the $2 k_{F}$ nesting would cause an incommensurate periodicity close to " $3 \sqrt{2}$," the interplay of the bulk electronic states and the strong $q$ dependence of the electron-phonon coupling is supposed to drive the system into the long-periodicity commensurate structure at $q$ close to $2 k_{F} / 3$. Upon the increase of In coverage, the shape and the size of the Fermi surface constituted by the $S_{1}$ resonance should change gradually. Since the electron-phonon coupling measured by the maximum band gap is rather large, $\Delta \sim 0.5 \mathrm{eV}$, in this system, ${ }^{2}$ the Fermi surface effect should have predominant, if not only, importance in determining the surface periodicity. For the systems with such strong electron-phonon coupling as the present one, the "bare" electronic susceptibility, determined by the $2 k_{F}$ nesting effect, is modified by the strong $q$ dependence of the electron-phonon matrix elements which represent the real-space effect such as the actual arrangement of atoms and shape of the wave functions involved. As a consequence, seemingly dissimilar surface structures will be realized upon a slight variation of the shape of the Fermi surface. Thus we suppose that the variety of structures in this system is predominantly determined by the Fermi surface topography. ${ }^{58}$ Clearly, this hypothesis should be verified rigorously. In particular, the detailed information on the evolution of $2 \mathrm{D}$ Fermi surface ${ }^{50}$ together with first-principles calculations should have great importance.

Surface resonance bands in general should exhibit the symmetry of the surface Brillouin zone. While a variety of long-range structures are observed in the present system, the band backfolding at surface Brillouin zone boundaries is observed only in the limited cases. Our photoemission data show that the resonance band described in the extended zone scheme gives the most strong photoemission intensity. The bands defined due to the reduced zone scheme give measurable intensity when it is backfolded according to the (1 $\times 1)$ periodicity. The band backfolding according to the superstructure periodicities, however, is not observed with a few exceptions including that due to the $(9 \sqrt{2} \times 2 \sqrt{2}) R 45^{\circ}$ periodicity (see Fig. 2 of Ref. 2). The insensitivity of photoemission to the surface superstructures is addressed to the matrix-element effect in the photoemission from crystals and should be accounted for by the photoemission structure factor. ${ }^{59,60}$

The In-induced surface resonance bands show different dispersion depending on the growth temperature, while the binding energy at the $\bar{X}$ symmetry point is almost the same when compared at the same local coverage. As is shown in Fig. 14(d), the $S_{1}$ band has dispersion similar to the $\mathrm{Cu} 4 s p$ band around $\bar{X}$. Upon deposition at $300 \mathrm{~K}$, In atoms are placed in close registry with $\mathrm{Cu}(001)-(1 \times 1)$ lattice and form ordered structures which are understood as periodically modulated $c(2 \times 2)$ lattices. Hence the dispersions measured at $300 \mathrm{~K}$ are ascribed to that of the interfacial states between $\mathrm{Cu}(001)-(1 \times 1)$ and the first In layer with $c(2 \times 2)$-like lattice. On the other hand, In atoms deposited at $110 \mathrm{~K}$ form 
islands with a $c(3 \sqrt{2} \times \sqrt{2}) R 45^{\circ}$ structure. An STM experiment revealed that the $c(3 \sqrt{2} \times \sqrt{2}) R 45^{\circ}$ structure consists of parallel rows of close-packed In atoms running along the [310] direction (Fig. 8). Thus the flat dispersion observed for In layers at $110 \mathrm{~K}$ is ascribed to the highly anisotropic interfacial structure. While the binding energies at high symmetry points in $k$ space are comparable with those for the structurally highly matched interface formed at $300 \mathrm{~K}$, the dispersion should be affected by anisotropic, poorly matched interface structure formed at $110 \mathrm{~K}$. This might have relevance to the fact that the structures formed at $110 \mathrm{~K}$ are metastable and, upon annealing, are transformed irreversibly to the same structures as those formed by deposition at $300 \mathrm{~K}$.

\section{SUMMARY}

We have studied the growth mode and the structure of the $\mathrm{In} / \mathrm{Cu}(001)$ system at deposition temperatures from 110 to $300 \mathrm{~K}$. Upon In deposition at $110-200 \mathrm{~K}$, the first layer grows via $2 \mathrm{D}$ island formation. The next layer seems to grow in the step-flow mode. The $c(3 \sqrt{2} \times \sqrt{2}) R 45^{\circ}$ and $(5 \sqrt{2}$ $\times 5 \sqrt{2}) R 45^{\circ}$ phases are formed successively. Upon In deposition at $300 \mathrm{~K}$, In initially forms surface alloy confined in the topmost substrate layer. Upon further deposition, a dealloying transition takes place around $0.26-0.35$ ML. Further In deposition results in the formation of three ordered phases: $(9 \sqrt{2} \times 2 \sqrt{2}) R 45^{\circ}, c(4 \times 4)$, and $(\sqrt{20}$ $\times \sqrt{20}) R 63.4^{\circ}+p(2 \times 2)$. The $(9 \sqrt{2} \times 2 \sqrt{2}) R 45^{\circ}$ and $c(4$ $\times 4)$ phases transform reversibly to $c(2 \times 2)$ and $p(2 \times 2)$, respectively, upon heating. The $(\sqrt{20} \times \sqrt{20}) R 63.4^{\circ}+p(2$ $\times 2)$ phase also transforms to $p(2 \times 2)$ upon heating, but in this case In coverage gradually decreases due to the desorption and/or dissolution.

A small amount of In atoms is known to have a strong surfactant effect in the $\mathrm{Cu} / \mathrm{Cu}(001)$ homoepitaxial growth. ${ }^{61}$ The effect is reported to depend strongly on the temperature, which was assumed to be due to the different adsorption sites of In on $\mathrm{Cu}(001)$. The present work serves a direct evidence for the spatial distribution of In atoms on $\mathrm{Cu}(001)$ at different temperatures and is believed to shed light onto the microscopic mechanism of the surfactant effect.

We observed surface resonance bands, $S_{1}$ and $S_{1}^{\prime}$, upon In deposition on $\mathrm{Cu}(001)$ at 300 and $110 \mathrm{~K}$, respectively. We assigned these surface resonances to the In $5 s p-\mathrm{Cu} 4 s p$ bonding states. These interfacial states may also be understood as split-off bands from $\mathrm{Cu} 4 s p$ due to the lowering of surface potential induced by the removal of excess surface charge by the In overlayers. The dispersion of the interfacial states is found to be different depending on the structure of the interfacial In layer.

The origin of a variety of ordered phases and transitions between them in this simple adsorption system, an $s p$ metal on an $s p$ metal surface, are quite intriguing. As one of the transitions has recently been shown to be due to the coupled effect of the electron-phonon interaction and the Fermi surface nesting, the role of surface electronic states in the ordering and transitions should be pursued. The account of this problem with respect to the valence electronic structure will be reported elsewhere. ${ }^{50}$
*Corresponding author. Email address: aruga@kuchem.kyotou.ac.jp

${ }^{1}$ Electronic Surface and Interface States on Metallic Systems, edited by E. Bertel and M. Donath (World Scientific, Singapore, 1994).

${ }^{2}$ T. Nakagawa, G. I. Boishin, H. Fujioka, H. W. Yeom, I. Matsuda, N. Takagi, M. Nishijima, and T. Aruga, Phys. Rev. Lett. 86, 854 (2001)

${ }^{3}$ K. Swamy, A. Menzel, R. Beer, and E. Bertel, Phys. Rev. Lett. 86, 1299 (2001).

${ }^{4}$ N. W. Ashcroft and N. D. Mermin, Solid State Physics (Saunders College Publishing, San Diego, 1976).

${ }^{5} \mathrm{~N}$. Eustathopoulos and J-C. Joud, in Current Topics in Materials Science, edited by E. Kaldis (North Holland, Amsterdam, 1980), Vol. 4.

${ }^{6}$ M. Hansen, Constitution of Binary Alloys (McGraw-Hill, New York, 1958).

${ }^{7}$ D. D. Chambliss and S. Chiang, Surf. Sci. 264, L187 (1992).

${ }^{8}$ L. P. Nielsen, F. Besenbacher, L. Stensgaard, E. Lægsgaard, C. Engdahl, P. Stoltze, and J. K. Nørskov, Phys. Rev. Lett. 74, 1159 (1995).

${ }^{9}$ C. Nagl, E. Platzgummer, O. Haller, M. Schmid, and P. Varga, Surf. Sci. 331-333, 831 (1995).

${ }^{10}$ S. Rpbert, S. Gauthier, F. Bocquet, S. Rousset, J. L. Duvault, and J. Klein, Surf. Sci. 350, 136 (1996).

${ }^{11}$ P. W. Murray, L. Stensgaard, E. Lægsgaard, and F. Besenbacher, Surf. Sci. 365, 591 (1996).
${ }^{12}$ P. T. Sprunger, E. Lægsgaard, and F. Besenbacher, Phys. Rev. B 54, 8163 (1996).

${ }^{13}$ S. D. Kevan, Surf. Sci. 178, 229 (1986).

${ }^{14}$ S.- $\AA$. Lindgren and L. Walldén, Phys. Rev. B 38, 3060 (1988).

${ }^{15}$ G. M. Watson, P. A. Bruhwiler, E. W. Plummer, H.-J. Sagner, and K.-H. Frank, Phys. Rev. Lett. 65, 468 (1990); G. M. Watson, P. A. Bruhwiler, H.-J. Sagner, K.-H. Frank, and E. W. Plummer, Phys. Rev. B 50, 17678 (1994).

${ }^{16}$ R. Fasel, P. Aebi, R. G. Agostino, L. Schlapbach, and J. Osterwalder, Phys. Rev. B 54, 5893 (1996).

${ }^{17}$ A. Carlsson, D. Claesson, G. Katrich, S.-A․ Lindgren, and L. Walldén, Phys. Rev. B 57, 13192 (1998).

${ }^{18}$ P. Heimann, J. Hermanson, H. Miosga, and H. Neddermeyer, Phys. Rev. B 20, 3059 (1979); Phys. Rev. Lett. 42, 1782 (1979).

${ }^{19}$ J. R. Smith, J. G. Gay, and F. J. Arlinghaus, Phys. Rev. B 21, 2201 (1980).

${ }^{20}$ A. Euceda, D. M. Bylander, L. Kleinman, and K. Mednick, Phys. Rev. B 27, 659 (1983).

${ }^{21}$ R. G. Jordan, G. Y. Guo, and L. R. Masliah, Solid State Commun. 99, 73 (1996).

${ }^{22}$ S. D. Kevan, Phys. Rev. B 28, 2268 (1983).

${ }^{23}$ R. van Gastel, E. Somfai, S. B. van Albada, W. van Saarloos, and J. W. M. Frenken, Phys. Rev. Lett. 86, 1562 (2001).

${ }^{24}$ C. Chen, T. T. Tsong, L. Zhang, and Z. Yu, Phys. Rev. B 46, 7803 (1992).

${ }^{25}$ M. Jiang, Y.-J. Zhao, and P-L. Cao, Phys. Rev. B 57, 10054 (1998). 
${ }^{26}$ M. Breeman and D. O. Boerma, Phys. Rev. B 46, 1703 (1992).

${ }^{27}$ M. Schmid, H. Stadler, and P. Varga, Phys. Rev. Lett. 70, 1441 (1993).

${ }^{28}$ A. Davies, J. A. Stroscio, D. T. Pierce, and R. J. Celotta, Phys. Rev. Lett. 76, 4175 (1996).

${ }^{29}$ T. Jung, Y. W. Mo, and F. J. Himpsel, Phys. Rev. Lett. 74, 1641 (1995).

${ }^{30}$ J. Peisner, P. Roboz, and P. B. Barna, Phys. Status Solidi A 4, K187 (1971).

${ }^{31}$ J. Topping, Proc. R. Soc. London, Ser. A 114, 67 (1927).

${ }^{32}$ A. R. Miller, Proc. Cambridge Philos. Soc. 42, 292 (1946).

${ }^{33}$ T. Aruga and Y. Murata, Prog. Surf. Sci. 31, 61 (1989).

${ }^{34}$ L. Schmidt and R. Gomer, J. Chem. Phys. 45, 1605 (1966).

${ }^{35}$ R. L. Gerlach and T. N. Rhodin, Surf. Sci. 19, 403 (1970).

${ }^{36}$ S. Andersson and U. Jostell, Surf. Sci. 46, 625 (1974).

${ }^{37}$ S. B. Lee, M. Weiss, and G. Ertl, Surf. Sci. 108, 357 (1981).

${ }^{38}$ A. G. Naumovets and Yu. S. Vedula, Surf. Sci. Rep. 4, 365 (1984).

${ }^{39}$ CRC Handbook of Chemistry and Physics, edited by D. R. Lide et al. (CRC Press, Boca Raton, FL, 1992).

${ }^{40}$ F. Theilmann, R. Matzdorf, and A. Goldmann, Solid State Commun. 72, 837 (1997); Surf. Sci. 387, 127 (1997).

${ }^{41}$ Wei Li, J.-S. Lin, M. Karimi, C. Moses, and G. Vidali, Appl. Surf. Sci. 48/49, 160 (1991).

${ }^{42}$ F. Bocquet and S. Gauthier, Surf. Sci. 416, 1 (1998).

${ }^{43}$ F. Delamare and G. E. Rhead, Surf. Sci. 35, 172 (1973).

${ }^{44}$ E. D. Hansen, T. Miller, and T.-C. Chiang, Phys. Rev. Lett. 78, 2807 (1997).

${ }^{45}$ D. M. Riffe, G. K. Wertheim, D. N. E. Buchanan, and P. H. Citrin, Phys. Rev. B 45, 6216 (1992).
${ }^{46}$ S. Doniach and M. Šunjić, J. Phys. C 3, 285 (1970).

${ }^{47}$ G. K. Wertheim and S. B. Dicenzo, J. Electron Spectrosc. Relat. Phenom. 37, 57 (1985); J. J. Joyce, M. Del Giudice, and J. H. Weaver, et al. 49, 31 (1989).

${ }^{48}$ S. I. Simak, U. Häussermann, R. Ahuja, S. Lidin, and B. Johansson, Phys. Rev. Lett. 85, 142 (2000).

${ }^{49}$ T. Abukawa, M. Sasaki, F. Hisamatsu, T. Kinoshita, A. Kakizaki, T. Goto, and S. Kono, J. Electron Spectrosc. Relat. Phenom. 80, 233 (1996).

${ }^{50}$ T. Nakagawa, H. Okuyama, M. Nishijima, and T. Aruga (unpublished).

51 T. Klas, R. Fink, G. Krausch, R. Platzer, J. Voigt, R. Wesche, and G. Schatz, Surf. Sci. 216, 270 (1989).

${ }^{52}$ R. Fink, G. Krausch, B. Luckscheiter, R. Platzer, U. Wöhrmann, and G. Schatz, Phys. Rev. Lett. 70, 2455 (1993).

${ }^{53}$ M. F. Roşu, F. Pleiter, and L. Niesen, Phys. Rev. B 63, 165425 (2001).

${ }^{54}$ T. Flores, M. Hansen, and M. Wutting, Surf. Sci. 279, 251 (1992).

${ }^{55}$ M. Breeman and D. O. Boerma, Surf. Sci. 287/288, 881 (1993).

${ }^{56}$ Clinton De W. Van Siclen, Phys. Rev. B 51, 7796 (1995).

${ }^{57}$ H. L. Meyerheim, H. Zajonz, W. Moritz, and I. K. Robinson, Surf. Sci. 381, L551 (1997).

${ }^{58}$ T. Aruga, J. Phys.: Condens. Matter (to be published).

${ }^{59}$ H. Daimon, S. Imada, H. Nishimoto, and S. Suga, J. Electron Spectrosc. Relat. Phenom. 76, 487 (1995).

${ }^{60}$ E. L. Shirley, L. J. Terminello, A. Santoni, and F. J. Himpsel, Phys. Rev. B 51, 13614 (1995).

${ }^{61}$ H. A. van der Vegt, M. Breeman, S. Ferrer, V. H. Etgens, X. Torrelles, P. Fajardo, and E. Vlieg, Phys. Rev. B 51, 14806 (1995). 